\title{
Measurement of the X-ray computed tomography instrument geometry by minimization of reprojection errors-implementation on simulated data
}

\author{
Massimiliano Ferrucci ${ }^{1,2}$, Petr Heřmánek ${ }^{3}$, Evelina Ametova ${ }^{1}$, Simone Carmignato ${ }^{3}$, Wim \\ Dewulf $^{1}$
${ }^{1}$ Mechanical Engineering Department, KU Leuven, 3000 Leuven (Belgium)
${ }^{2}$ Engineering Measurement Division, National Physical Laboratory, TW11 0LW Teddington (UK)

${ }^{3}$ Department of Management and Engineering, University of Padova, 36100 Vicenza (Italy)

Corresponding author: massimiliano.ferrucci@kuleuven.be, +32 476931594

\section{Abstract}

The X-ray computed tomography (CT) measurement process suffers from a variety of error sources including geometrical misalignments of the CT imaging components: X-ray source focal spot, rotation stage, and X-ray detector. Effective correction of these misalignments demands accurate measurement of the instrument geometry. A common method to measure geometrical parameters involves radiographically imaging a reference object consisting of several high X-ray absorption spheres. The resulting projection images are used to solve the CT geometry of the instrument. Geometrical parameters can be solved either analytically or by minimization of reprojection errors. In this study, the minimization technique is chosen over analytical methods due to the relative versatility in its practical implementation. Various precautions must be taken when using minimization techniques and these are discussed. Errors in the data acquisition and subsequent minimization procedure introduce errors in the measured geometry and are investigated here. The geometrical measurement procedure is performed on simulated data using the Computed Tomography Calibration Tube $\left(\mathrm{CT}^{2}\right)$, a reference object that was designed to reduce sphere overlaps in the projected images while at the same time broadening the distribution of projected spheres across the detector area. It should be noted, however, that the proposed procedure can be implemented with any reference object consisting of high $\mathrm{X}$-ray absorption spheres, provided the coordinate positions of the sphere centers in a local frame are known. Residual errors between the true (simulated) and measured geometrical parameters are shown to be negligible from tomographic reconstruction of datasets acquired in the presence of these residual errors.

Keywords: X-ray computed tomography, dimensional metrology, instrument alignment

\section{Introduction}

X-ray computed tomography (CT) is a promising solution for non-destructive coordinate measurement of internal and external features. However, CT instruments have not yet reached measurement maturity due to a lack of standardized procedures for evaluating uncertainty and, ultimately, achieving traceable CT measurements. Uncertainty is a result of various influence factors in the measurement procedure, including errors inherent to the instrument and user-induced errors. The GUM method (named after the document in which it is described: the Guide to the Expression of Uncertainty in Measurement [1]) for evaluating measurement uncertainty demands that each source of error be determined and its quantity propagated to uncertainty in the measurement result. Physical misalignments in the CT 
instrument can contribute to measurement uncertainty and their measurement is the focus of this research. This study pertains to cone-beam CT instruments but its findings are also relevant for other $\mathrm{CT}$ architectures given the appropriate modifications.

The accuracy of CT measurements relies on the accurate knowledge of the relative position and orientation of the three major instrument components: X-ray source focal spot, object rotation axis, and X-ray detector. This spatial information is used in the back projection step of the tomographic reconstruction algorithm to produce a three-dimensional attenuation map of the measurement volume, which is defined as a three-dimensional array of voxels. For each angular position of the rotation stage, a two-dimensional radiographic image of the measurement volume is acquired. The measured X-ray intensity at each pixel is back projected as a linear trajectory from the corresponding pixel position to the X-ray focal spot. Each trajectory traces a path through the measurement volume thereby intersecting a series of voxels along that path (figure 1). Attenuation values for each voxel are calculated from the collection of radiographic intensities, the back projected trajectories of which intersect the voxel. If the geometry used in the back projection step is not accurately known, the attenuation values for each voxel will be erroneously calculated and subsequent measurements performed on the reconstructed volume will also include errors. It is therefore critical that the geometry of the CT instrument be known accurately to ensure correct tomographic reconstruction of scanned parts [2].

Conventional tomographic reconstruction algorithms typically assume nominal alignment of the CT components (see section 2 for a detailed description of the aligned CT geometry). To ensure accurate reconstruction using these algorithms, any misalignment in the instrument should be adjusted to bring the instrument to its aligned state. Users of commercial CT instruments typically rely on instrument manufacturers to measure and adjust any misalignments in the CT components. The scope of this paper is to present a method with which users can measure the geometry of a cone-beam CT instrument. Various methods to measure the CT geometry have been presented in the literature and are summarized in [3]; however, calibration of the geometry per the metrological definition has not been demonstrated. In the absence of proven calibration, measured geometrical parameter values are simply considered estimates. This study takes a step towards achieving calibration by performing a metrological investigation of the geometrical measurement procedure.

Estimation methods typically consist of solving a set of geometrical parameters by radiographically imaging a reference object and analyzing the acquired projection data. The most common type of reference object consists of several high X-ray absorption spherical markers. Calibration of the CT geometry demands the comparison of the measured geometrical parameters to a traceable reference. It is therefore critical that the reference features in the imaged object, in this case the three-dimensional coordinate positions of the sphere centers, be calibrated. Furthermore, error sources in the geometrical calibration procedure must be quantified and propagated to uncertainty in the geometrical parameters.

In this study, we propose a method to measure the geometry of a cone-beam CT instrument using a traceable reference object. Geometrical parameters are determined by minimizing reprojection errors, i.e. the difference between observed and modelled projection data of the reference object. Practical considerations are provided for the analysis of radiographic data and for the robust implementation of the minimization technique. The proposed method is 
applied on simulated radiographic data of the Computed Tomography Calibration Tube $\left(\mathrm{CT}^{2}\right)$ reference object [4]. It should be noted that the geometrical measurement procedure presented here can be implemented with any reference object consisting of high-absorption spheres provided the positions of the sphere centers in a local coordinate frame are known, e.g. from $\mathrm{CMM}$ measurements. Uncertainty in the reference object features and error motions of the rotation stage are included to approximate the simulated environment to what can be expected in experimental data (see section 3). The application of the geometrical measurement procedure to simulated data allows us to compare the measured geometrical parameter values to the actual simulated values. Furthermore, application of the proposed method on simulated data is a critical first step to validate the implementation on experimental data. For this reason, discussions are provided throughout this paper to address this discrepancy and suggestions are made for eventually implementing the method on the measurement of experimental CT geometries. Finally, a discussion on error sources in the proposed method is provided in the context of future work on assessing uncertainty in the measured geometrical parameters, which is required for calibration of the CT instrument geometry.

\section{Geometry of a cone-beam CT instrument and its measurement}

\subsection{CT instrument geometry}

The geometry of a cone-beam CT instrument is defined by the relative position and orientation of X-ray source focal spot, axis of object rotation (AOR), and detector. Since a reference object is used in the geometrical estimation, its position and orientation must also be defined with respect to the components of the $\mathrm{CT}$ instrument. The diagram in figure 2, which illustrates a typical cone-beam CT instrument, supplements the following description of the coordinate convention. A right-handed global Cartesian coordinate system is fixed with its origin at the $\mathrm{X}$-ray source focal spot $\mathrm{S}$. The $\mathrm{Y}$ axis is parallel to the AOR, while the $\mathrm{Z}$ axis is coincident with the line from $S$ that intersects the AOR orthogonally. The $X$ axis subsequently follows the right-hand screw rule, where $(X \times Y)=Z$. In an aligned instrument, the detector rows are parallel to the global $X$ axis, while the detector columns are parallel to the $Y$ axis. The $Z$ axis ideally intersects the detector at its geometrical center. The $\mathrm{U}$ and $\mathrm{V}$ axes of the detector coordinate frame correspond to the indexing axes for the detector column and rows, respectively.

The location of the AOR is given by the coordinate position of its intersection with the $\mathrm{Z}$ axis (also known as the center of rotation) $\mathbf{R}=\left(x_{\mathrm{R}}, y_{\mathrm{R}}, z_{\mathrm{R}}\right)$. By definition, the point $\mathbf{R}$ is located on the $\mathrm{Z}$ axis, therefore $x_{\mathrm{R}}=y_{\mathrm{R}}=0$ and the position of the AOR can be parameterized by the single coordinate $z_{r}$, that is $\mathbf{R}=\left(0,0, z_{\mathrm{R}}\right)$. The orientation of the AOR is given by the unit vector $\hat{\boldsymbol{r}}=(0,1,0)$. Rotation of the object is parameterized by the angle $\alpha$, the positivity of which follows the right hand screw rule with respect to $\hat{\boldsymbol{r}}$.

The position of the detector center is parameterized by the point $\mathbf{D}=\left(x_{\mathrm{D}}, y_{\mathrm{D}}, z_{\mathrm{D}}\right)$. Detector orientation is defined by three extrinsic rotations performed about local axes that are parallel to the axes of the global coordinate system and whose origin is the detector center: tilt $\theta$ about the local $\mathrm{X}$-axis, slant $\phi$ about the local $\mathrm{Y}$-axis, and skew $\eta$ about the local $Z$-axis. The positive direction of rotation is given by the right-hand screw rule. Rotations are applied in the following sequence: $\eta, \phi$, then $\theta$. The rotation convention was chosen to correspond to the convention in Scorpius $\mathrm{XLab}^{\circledR}$ simulation software (Fraunhofer IIS, Germany). 
The parameterization of the reference object in the CT geometry is given by the position of its local origin and the orientation of its local axes with respect to the global origin and coordinate axes, respectively, at the $\alpha=0^{\circ}$ position of the rotation stage. The position of the local origin in the global frame is given by the point $\mathbf{P}=\left(x_{\mathrm{P}}, y_{\mathrm{P}}, z_{\mathrm{P}}\right)$ and the orientation of the local axes is given by three extrinsic rotations, performed sequentially in the order (1) $\rho_{\mathrm{Y}},(2) \rho_{\mathrm{Z}}$, and (3) $\rho_{\mathrm{X}}$. The reference object rotation sequence was chosen to correspond to the sequence in the simulation software. These parameters are considered nuisance parameters as they do not describe the CT geometry, yet are necessary for solving the minimization problem. Furthermore, these nuisance parameters eliminate the need to know precisely the position and orientation of the reference object in the global coordinate frame. The CT geometry can therefore be defined by 13 geometrical parameters: 7 instrument

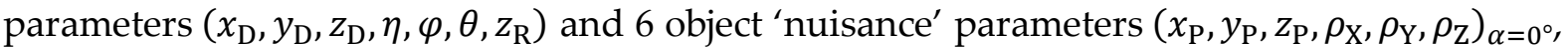
which are also summarized in table 1.

Table 1. Geometrical parameters for CT instrument and reference object.

\begin{tabular}{|l|l|c|}
\hline Component & Feature & Parameters \\
\hline X-ray focal spot & Position & $\mathbf{S}=(0,0,0)$ \\
\hline AOR & Position & $\mathbf{R}=\left(0,0, z_{\mathrm{R}}\right)$ \\
\hline AOR & Orientation & $\hat{\boldsymbol{r}}=(0,1,0)$ \\
\hline Detector & Position & $\mathbf{D}=\left(x_{\mathrm{D}}, y_{\mathrm{D}}, z_{\mathrm{D}}\right)$ \\
\hline Detector & Orientation & $(\eta, \varphi, \theta)$ \\
\hline Reference object & Position & $\mathbf{P}=\left(x_{\mathrm{P}}, y_{\mathrm{P}}, z_{\mathrm{P}}\right)_{\alpha=0^{\circ}}$ \\
\hline Reference object & Orientation & $\left(\rho_{\mathrm{X}}, \rho_{\mathrm{Y}}, \rho_{\mathrm{Z}}\right)_{\alpha=0^{\circ}}$ \\
\hline
\end{tabular}

The geometry of a CT instrument can be determined by imaging a dedicated reference object at $\mathrm{N}$ angular positions of the rotation stage from 0 to $360^{\circ}$; in this study, angular positions are equally spaced, though it is not a requirement. The rotation angle of each rotation position $n=1,2, \ldots, \mathrm{N}$ is given by $\alpha_{n}$. The acquired projection images can then be used to estimate the CT imaging geometry by way of dedicated analyses [3]. Most commonly used reference objects consist of several high X-ray absorption spherical markers distributed in a particular arrangement on a cylindrical structure. This structure is typically made from a relatively low absorption material to ensure high contrast of the projected markers in the radiographs. Each sphere $m$, where $m=1,2, \ldots, \mathrm{M}$ and $\mathrm{M}$ is the total number of spheres, is defined by the threedimensional coordinate position of its center $(x, y, z)_{m}^{\text {local }}$ in the local (object) coordinate frame. The sphere center coordinates in the global coordinate frame and at the rotation position $\alpha=$ $0^{\circ}$ of the rotation stage $(x, y, z) \underset{m}{\text { global }}$ are given by applying the three rotations $\left(\rho_{\mathrm{X}}, \rho_{\mathrm{Y}}, \rho_{\mathrm{Z}}\right)$ and the three translations $\left(x_{\mathrm{P}}, y_{\mathrm{P}}, z_{\mathrm{P}}\right)$ to the sphere center coordinates in the local coordinate frame. This operation is conceptualized in equation (1) below.

$$
(x, y, z)_{m, \alpha=0^{\circ}}^{\text {global }}=f\left(x_{m}^{\text {local }}, y_{m}^{\text {local }}, z_{m}^{\text {local }}, \rho_{\mathrm{X}}, \rho_{\mathrm{Y}}, \rho_{\mathrm{Z}}, x_{\mathrm{P}}, y_{\mathrm{P}}, z_{\mathrm{P}}, \alpha=0^{\circ}\right)
$$

In the projection image of the reference object, the set of M projected sphere center coordinates $\left(u_{\text {obs }}, v_{\text {obs }}\right)_{m}$-henceforth referred to as center projection coordinates-are determined utilizing the image analysis procedure described in section 4 . 
For each angular position $n$ of the rotation stage, the global sphere center coordinates are rotated by the corresponding angle $\alpha_{n}$, where $\alpha_{n}=n\left(\frac{360^{\circ}}{\mathrm{N}}\right)$ for equally spaced rotation positions, thereby providing a new set of sphere center coordinates $(x, y, z)_{m, n}^{\text {global }}$. Analysis of the newly acquired projection image provides estimates of the corresponding set of center projection coordinates $\left(u_{\mathrm{obs}}, v_{\mathrm{obs}}\right)_{m, n}$ (figure 3 ). The complete scan of the reference object and subsequent image analysis provides a set of $\mathrm{M} \times \mathrm{N}$ sphere center coordinates $(x, y, z)_{m, n}^{\text {global }}$ and a corresponding set of $\mathrm{M} \times \mathrm{N}$ center projection coordinates $\left(u_{\mathrm{obs}}, v_{\mathrm{obs}}\right)_{m, n}$.

Geometrical parameters can be determined analytically, that is by solving equations relating the sphere center coordinates $(x, y, z)_{m, n}$ in the global coordinate frame and observed center projection coordinates $\left(u_{\mathrm{obs}}, v_{\mathrm{obs}}\right)_{m, n}$ to the set of instrument parameters $p_{i}$, where $i=$ $1,2, \ldots, 7$. Analytical methods typically rely on the precise alignment of the reference object, i.e. on the prior knowledge of the 6 reference object nuisance parameters, thereby making the accuracy of the solved parameters dependent on the exactness of this alignment [3]. This requirement makes analytical methods unwieldy.

\subsection{Measurement by minimization of reprojection errors}

Minimization methods consist of finding the set of modelled geometrical parameters that minimize the least-squares error between modelled and observed center projection coordinates, known as the reprojection error [2,5]. A ray-tracing model is used to generate the center projection coordinates for a reference object with known sphere positions given an initial set of geometrical parameter values. The set of modelled center projection coordinates $\left(u_{\text {mod }}, v_{\text {mod }}\right)_{m, n}$ are compared to the set of observed center projection coordinates $\left(u_{\mathrm{obs}}, v_{\mathrm{obs}}\right)_{m, n}$ from analysis of acquired radiographs. The sum of squared residuals (SSR) between modelled and observed center projection coordinates, shown in equation (2), is used as the objective function to be minimized. It should be noted that the objective function can take other algebraic forms, for example keeping the $u$ and $v$ components of the center projection residuals separate prior to summing instead of summing in quadrature. The implications of other objective functions on the performance of the minimization is a topic of further research.

$$
\operatorname{SSR}=\sum_{n=1}^{\mathrm{N}} \sum_{m=1}^{\mathrm{M}}\left(\sqrt{\left(u_{\bmod }(m, n)-u_{\mathrm{obs}}(m, n)\right)^{2}+\left(v_{\bmod }(m, n)-v_{\mathrm{obs}}(m, n)\right)^{2}}\right)^{2}
$$

The fundamental principle of minimization methods is to iteratively adapt-or 'solve' - the geometrical parameters of the ray-tracing model to minimize the reprojection error. If the minimization procedure is implemented correctly, the solved parameters that provide the lowest reprojection error should correspond to the parameters of the test instrument with which the observed coordinates were acquired. A shortcoming of minimization methods is the possibility of solving to a local minimum, which would provide an erroneous set of solved parameters. Global optimization tool Global Search [6] is used to reduce the occurrence of solving to local minima and consists of repeating the minimization procedure under different initial values of the solvable parameters.

\subsection{Reference object}


The reference object used in this study is based on the prototype from [4], in which 49 high Xray absorption (steel) spheres of $2.5 \mathrm{~mm}$ diameter are arranged in four helices, each performing one full helical turn along the outer circumference of a carbon fiber cylindrical support. Spheres at the top and bottom of the helices perform a full circular trajectory to allow static determination of the instrument geometry, as described in [8]. In this study, only the spheres are simulated (i.e. the carbon fiber structure is not included when generating radiographs) to allow the focus of the investigation to be on the geometrical measurement procedure. Each sphere is defined by the coordinate position of its center in a local coordinate frame (figure 4). In this study, the sphere center coordinate positions are given by their nominal values plus random perturbations to account for uncertainty in calibrated sphere coordinates expected in experimental implementation (see section 3 ).

The coordinate position of each sphere center both in the global frame and on the projection image constitute corresponding data points. Overlaps between projected spheres can occur, particularly for reference objects with a relatively large number of spheres. If not properly considered, these overlaps can introduce errors in the estimation of the center projections and, ultimately, can compromise the effective implementation of geometrical estimation. The $\mathrm{CT}^{2}$ was designed to reduce sphere overlaps in the projections while maintaining the number of spheres that was deemed optimal in [9]. For a sample data set, the $\mathrm{CT}^{2}$ object resulted in less than $1 \%$ of data points having overlaps.

\section{Simulation of radiographic data acquisition}

Scorpius $\mathrm{XLab}{ }^{\circledR}$ (Fraunhofer IIS, Germany) is used for generating radiographs of the reference object. The unvaried settings used in all simulated acquisitions are provided in table 2. Acquisition of the reference object is simulated under $s=1,2, \ldots, 10$ instrument misalignments, and various orientations and positions of the reference object, summarized in Table 3 and Table 4, respectively.

Table 2. Parameters in the simulated acquisition of radiographs.

\begin{tabular}{l|l} 
Parameter & Value \\
\hline Acceleration voltage & $100 \mathrm{kV}$ \\
Filament current & $0.1 \mathrm{~mA}$ \\
Number of projections & $720\left(\right.$ from $0^{\circ}$ to $359.5^{\circ}$ in increments of $\left.0.5^{\circ}\right)$ \\
Pixel size & $0.2 \mathrm{~mm}$ \\
Detector size & $2000 \times 2000$ pixels
\end{tabular}

To approximate the effects of uncertainty in the reference measurements of sphere center coordinates, e.g. by CMM, in experimental implementation, the set of $m$ nominal (CAD) sphere center coordinates $(x, y, z)_{m}^{\text {nominal }}$ are perturbed by $(\Delta x, \Delta y, \Delta z)_{m, s}$, which are randomly sampled (details in section 3.2) and applied separately for each simulation s.

$$
(x, y, z)_{m, s}^{\text {perturbed }}=(x, y, z)_{m}^{\text {nominal }}+(\Delta x, \Delta y, \Delta z)_{m, s}
$$

The sphere center coordinates in the global coordinate frame at the first angular position of the sample stage are calculated as follows. 


$$
(x, y, z)_{m, s, \alpha=0^{\circ}}^{\text {global }}=\left[\rho_{s}\right](x, y, z)_{m, s}^{\text {perturbed }}+\left(x_{\mathrm{P}}, y_{\mathrm{P}}, z_{\mathrm{P}}\right)_{s}
$$

where $\left[\rho_{s}\right]$ is the rotation matrix and $\left(x_{\mathrm{P}}, y_{\mathrm{P}}, z_{\mathrm{P}}\right)_{s}$ is the translation vector for orienting and positioning the local sphere center coordinates in the global coordinate frame. The global sphere center coordinate positions for each rotation position of the sample stage are given by applying the rotation matrix $\left[\alpha_{\text {actual }, s}(n)\right]$ to $(x, y, z)_{m, s, \alpha=0^{\circ}}^{\text {global }}$ as follows.

$$
(x, y, z)_{m, s, n}^{\text {rotated }}=\left[\alpha_{\text {actual }, s}(n)\right](x, y, z)_{m, s, \alpha=0^{\circ}}^{\text {global }}
$$

The actual rotation angles are calculated by applying indexing errors to the nominal indexed angles, as follows.

$$
\alpha_{\text {actual }, s}(n)=\alpha_{\text {index }}(n)+\delta_{s}(n)
$$

where $\alpha_{\text {index }}(n)=n\left(\frac{360^{\circ}}{\mathrm{N}}\right)$ are the $n=0,1, \ldots, \mathrm{N}$ nominal equally-spaced indexed rotation angles and $\delta_{s}(n)$ are indexing errors, detailed in section 3.3.

Tilt errors of the sample stage are applied as follows.

$$
(x, y, z)_{m, s, n}^{\text {tilt error }}=[\gamma(n)](x, y, z)_{m, s, n}^{\text {global }}
$$

where $[\gamma(n)]$ is the tilt error operator, described in section 3.3. Finally, radial error motions $\tau_{\mathrm{X}, s}(n)$ and $\tau_{\mathrm{Z}, S}(n)$, and axial error motion $\tau_{\mathrm{Y}, S}(n)$ are applied as follows.

$$
(x, y, z)_{m, s, n}^{\text {final }}=(x, y, z)_{m, s, n}^{\text {tilt error }}+\left(\tau_{\mathrm{X}}(n), \tau_{\mathrm{Y}}(n), \tau_{\mathrm{Z}}(n)\right)_{s}
$$

In order to simulate the acquisition of the reference object with rotation stage errors on Scorpius XLab, separate XML files for each rotation position $n$ are generated. The set of sphere center coordinates $(x, y, z)_{m, s, n}^{\mathrm{final}}$ are used to define the locations of each sphere $m$ within each XML file and for each simulation $s$. Blur and noise are added to the radiographs in MATLAB after generation by Scorpius XLab. Blur is applied as a two-dimensional smoothing kernel with standard deviation of 1 pixel, chosen to correspond to blur observed in similar radiographs acquired on an experimental CT instrument. After blurring, Poisson noise is added to approximate the expected Poisson variation in the photon output of the X-ray source.

\subsection{Simulated instrument parameters}

The misaligned instrument geometrical parameters for each simulation s are shown in table 3. The magnitude of each misalignment was randomly chosen from a uniform distribution, the intervals of which correspond to what could be realistically expected, but not readily noticeable, misalignments in CT instruments. The geometrical values in the first row of table 3 correspond to the definition in this study of an 'aligned' instrument. These values are used as the initial parameter values in the geometrical measurement procedure (see section 5.2) and correspond to the geometry assumed by the tomographic reconstruction algorithm in section 5.3. However, the simulated acquisition of the $\mathrm{CT}^{2}$ reference object and subsequent geometrical measurement are not performed under 'aligned' instrument geometry. For each simulation $s$, the position and orientation of the reference object at the $\alpha=0^{\circ}$ position is 
varied, as shown by the reference object geometrical parameters in table 4 . There are no requirements for reference object position and orientation in the geometrical measurement procedure, provided all spheres are in the detector field of view in all acquired radiographs.

Table 3. Simulated values for instrument geometrical parameters.

\begin{tabular}{c|ccccccc}
$\boldsymbol{s}$ & $\begin{array}{c}\boldsymbol{x}_{\mathbf{D}} \\
/ \mathbf{m m}\end{array}$ & $\begin{array}{c}\boldsymbol{y}_{\mathbf{D}} \\
/ \mathbf{m} \mathbf{m}\end{array}$ & $\begin{array}{c}\mathbf{z}_{\mathbf{D}} \\
/ \mathbf{m m}\end{array}$ & $\begin{array}{c}\mathbf{z}_{\mathbf{R}} \\
/ \mathbf{m m}\end{array}$ & $\begin{array}{c}\boldsymbol{\theta} \\
\boldsymbol{\rho}^{\circ}\end{array}$ & $\begin{array}{c}\boldsymbol{\varphi} \\
\boldsymbol{\circ}^{\circ}\end{array}$ & $\begin{array}{c}\boldsymbol{\eta} \\
\boldsymbol{\rho}^{\circ}\end{array}$ \\
\hline Aligned & 0 & 0 & -1177 & -400 & 0 & 0 & 0 \\
1 & 1.2590 & -1.3700 & -1175.4430 & -402.5450 & -0.6756 & -0.0989 & -0.7867 \\
2 & 1.6230 & 1.8820 & -1181.6430 & -402.6760 & 0.5886 & -0.8324 & 0.9238 \\
3 & -1.4920 & 1.8290 & -1173.5090 & -399.8150 & -0.3776 & -0.5420 & -0.9907 \\
4 & 1.6540 & -0.0590 & -1172.6600 & -398.3250 & 0.0571 & 0.8267 & 0.5498 \\
5 & 0.5290 & 1.2010 & -1175.2130 & -397.3960 & -0.6687 & -0.6952 & 0.6346 \\
6 & -1.6100 & -1.4330 & -1174.4230 & -402.2210 & 0.2040 & 0.6516 & 0.7374 \\
7 & -0.8860 & -0.3130 & -1174.5690 & -399.5870 & -0.4741 & 0.0767 & -0.8311 \\
8 & 0.1880 & 1.6630 & -1178.0780 & -400.1840 & 0.3082 & 0.9923 & -0.2004 \\
9 & 1.8300 & 1.1690 & -1175.4460 & -402.9290 & 0.3784 & -0.8436 & -0.4803 \\
10 & 1.8600 & 1.8380 & -1180.2880 & -400.9770 & 0.4963 & -0.1146 & 0.6001
\end{tabular}

Table 4. Simulated values for reference object, i.e. 'nuisance', geometrical parameters at $\alpha=$ $0^{\circ}$ rotation position.

\begin{tabular}{c|cccccc}
$\boldsymbol{S}$ & $\begin{array}{c}\boldsymbol{x}_{\mathbf{P}} \\
/ \mathbf{m m}\end{array}$ & $\begin{array}{c}\boldsymbol{y}_{\mathbf{P}} \\
/ \mathbf{m m}\end{array}$ & $\begin{array}{c}\mathbf{Z}_{\mathbf{P}} \\
/ \mathbf{m m}\end{array}$ & $\begin{array}{c}\boldsymbol{\rho}_{\mathbf{X}} \\
/{ }^{\circ}\end{array}$ & $\begin{array}{c}\boldsymbol{\rho}_{\mathbf{Y}} \\
\boldsymbol{}^{\circ}\end{array}$ & $\begin{array}{c}\boldsymbol{\rho}_{\mathbf{Z}} \\
\boldsymbol{}^{\circ}\end{array}$ \\
\hline 1 & 1.0051 & 1.3629 & -400.5934 & 0.4121 & -0.1225 & -0.4479 \\
2 & -0.9796 & -0.9829 & -398.6767 & -0.9363 & -0.2369 & 0.3594 \\
3 & 0.0238 & 1.2571 & -399.6589 & -0.4462 & 0.5310 & 0.3102 \\
4 & 0.7963 & -1.0259 & -399.8011 & -0.9077 & 0.5904 & -0.6748 \\
5 & 1.5636 & 1.7171 & -398.3312 & -0.8057 & -0.6263 & -0.7620 \\
6 & 1.8372 & -0.6001 & -400.8566 & 0.6469 & -0.0205 & -0.0033 \\
7 & 0.1889 & -1.2136 & -398.9712 & 0.3897 & -0.1088 & 0.9195 \\
8 & -1.4455 & -0.9957 & -398.9851 & -0.3658 & 0.2926 & -0.3192 \\
9 & -1.4028 & 0.4642 & -400.4782 & 0.9004 & 0.4187 & 0.1705 \\
10 & -0.9700 & -0.1068 & -399.7287 & -0.9311 & 0.5094 & -0.5524
\end{tabular}

\subsection{Perturbation of sphere center coordinates}

In experimental implementation, there is uncertainty in the calibrated sphere center coordinates. To account for this uncertainty, the nominal center coordinates of the simulated spheres are perturbed from their nominal values in the local coordinate frame by $\Delta x_{m, s}, \Delta y_{m, s}$, and $\Delta z_{m, s}$, the values of which are randomly sampled coordinate perturbations from normal distributions with zero mean and standard deviation $\sigma=0.603 \mu \mathrm{m}$. The uncertainty 
corresponds to the expected uncertainty in the calibration of sphere center coordinates by CMM. The perturbation is repeated separately for each simulation $s$.

\subsection{Rotation stage error motions}

Rotation stage error motions are applied as a combination of systematic and random perturbations to the sphere center coordinates in the simulated scans as a function of rotation stage position $n$. The modelled error motions are angular indexing error, axial error motion, radial error motion, and tilt error motion (figure 5). The function and magnitude of each modelled error motion correspond to the observed error functions and acceptance limits reported in the manufacturer control report of a Newport RVS80CC rotation stage employed in the $225 \mathrm{kV}$ CT instrument (Nikon MCT225) at the National Physical Laboratory. The control report did not provide specifications for axial error motion; the specification value for radial error motion was therefore also applied to set the axial error motion interval. The characteristics of the modelled error motions are described in table 5. Random error motions are sampled from uniform (square) distributions symmetrical about zero; the magnitude of the distributions are defined by the interval boundaries.

Angular indexing error $\delta_{s}(n)$ is an error between the indexed angle $\alpha_{\text {index }}(n)$ and the actual rotation stage angular position $\alpha_{\text {actual }}(n)$, and is modelled as a systematic first order harmonic component (corresponding to eccentricity between the center of the encoder grating and the axis of rotation) and a random component. Axial error motion $\tau_{Y, s}(n)$ is a translation of the stage along the axis of rotation. Translations of the rotation stage along the plane normal to the axis of rotation are known as radial error motions and are parameterized by two translations $\tau_{\mathrm{X}, S}(n)$ and $\tau_{\mathrm{Z}, \mathrm{s}}(n)$ along the respective axis directions. Tilt error motions, also known more informally as 'wobble', are non-zero order tilts of the rotation stage surface normal with respect to the axis of rotation; a constant (zero order) tilt of the rotation stage surface normal with respect to the axis of rotation is inconsequential to the quality of CT data. Two angles $\gamma(n)$ and $\xi_{s}(n)$ are used to parameterize tilt error motion (figure 6). $\gamma(n)$ is the magnitude of the tilt and consists of two systematic components: a half-order harmonic component and a $13^{\text {th }}$ order harmonic as a result of, among others, imperfect ball bearings between stage stator and rotor. $\xi_{s}(n)$ is the angle along the $\mathrm{XZ}$ plane denoting the direction of a unit vector about which the tilt error motions are applied. The tilt direction vector is assigned a starting angle $\xi_{0, \mathrm{~s}}$ randomly chosen between 0 to $2 \pi$, i.e. $\xi_{0, \mathrm{~s}}$ denotes the orientation of the tilt direction vector at $\alpha=0^{\circ}$. For each subsequent rotation position of the stage $\alpha_{\text {index }}(n)$, the tilt direction vector is rotated along the $\mathrm{XZ}$ plane, as expressed in equation (9). The center of rotation for tilt error motion is located $105 \mathrm{~mm}$ below the $\mathrm{Y}$ position of the local object origin and along the axis of rotation.

$$
\xi_{s}(n)=\xi_{0, s}+\alpha_{\text {index }}(n)
$$

Table 5. Functions and magnitudes for the modelled rotation stage errors. 'Random' denotes random sampling from a uniform distribution, the interval $[-a,+a]$ of which is given by the values in the corresponding magnitude column. The magnitude for harmonic error 
components corresponds to the maximum and minimum intensity of the corresponding waveform.

\begin{tabular}{l|c|c|c} 
Error source & Parameter & Sampling & Magnitude \\
\hline \multirow{2}{*}{ Indexing error } & $\delta_{1}(n)$ & First order harmonic & \pm 0.0027 degrees \\
& $\delta_{\mathrm{r}, s}(n)$ & Random & \pm 0.0003 degrees \\
Tilt error motion & $\gamma_{1 / 2}(n)$ & Half-order harmonic & $\pm 18 \mu$ radians \\
& $\gamma_{13}(n)$ & Thirteenth-order harmonic & $\pm 2 \mu$ radians \\
Radial error motion $(\mathrm{X})$ & $\tau_{\mathrm{X}, s}(n)$ & Random & $\pm 2 \mu \mathrm{m}$ \\
Radial error motion $(\mathrm{Z})$ & $\tau_{\mathrm{Z}, s}(n)$ & Random & $\pm 2 \mu \mathrm{m}$ \\
Axial error motion & $\tau_{\mathrm{Y}, s}(n)$ & Random & $\pm 2 \mu \mathrm{m}$
\end{tabular}

\section{Analyzing the projection data}

Analysis of the projection data consists of estimating the pixel coordinates of the center projections for all acquired radiographs. Three practical considerations are presented here, namely the definition of the center projection from the imaged sphere, automation of the analysis step by sphere tracking, and managing overlaps in the projected spheres.

\subsection{Estimating center projection coordinates}

Correctly determining the location of the center projection from the imaged sphere in the radiograph is not trivial and has been an ongoing topic of research [10-12]. In the absence of other objects in the field of view, a sphere is projected onto the detector as an elliptical disk [11]. Assuming a single-material sphere with uniform density, the intensity recorded by each pixel within the elliptical disk is proportional to the path length of the corresponding X-ray trajectory through the sphere. The longest path through a perfect sphere is the path that contains the sphere center; therefore, the projection point of the sphere center $\mathbf{P}$ is theoretically the position on the projected elliptical disk with a minimum intensity, i.e. highest X-ray attenuation [12].

In practice, however, effects such as image blur, noise, and photon depletion (complete X-ray absorption by objects) result in low image gradients in the area of lowest intensity. It is therefore difficult to accurately locate the sphere center projection from the intensity minimum. An alternative to estimating the coordinate positions of the projected spheres consists of using the center $\boldsymbol{e}$ of an ellipse fit to the outer edge of the projected disk. Detailed investigations in the use of this alternative method of estimation are provided in $[10,11]$. It was found in [11] that the true sphere center projection $\mathbf{P}$ lies on the major axis of the projected elliptical disk, albeit closer to the orthogonal projection of the source onto the detector (also known as the principal point, $\mathbf{O}$ ) than $\boldsymbol{e}$ (figure 7).

Deng et al. [12] propose an enhanced estimate $\boldsymbol{p}$ of the sphere center projection coordinates $\mathbf{P}$ by applying a correction to the fit ellipse center $\boldsymbol{e}$ along the ellipse major axis in the direction of the principal point. The correction is calculated from the lengths of the major and minor axes of the fit ellipse $L_{\text {major }}$ and $L_{\text {minor }}$, respectively, as shown in equation (10).

$$
\boldsymbol{p}=\boldsymbol{e}-\frac{L_{\text {major }}^{2}-L_{\text {minor }}^{2}}{4 \boldsymbol{e}}
$$


where $\boldsymbol{p}$ is the two-dimensional vector denoting the position of the corrected center projection coordinates on the detector, $\boldsymbol{e}$ is the two-dimensional vector denoting the center coordinates of the fit ellipse, and $L_{\text {major }}$ and $L_{\text {minor }}$ are the scalar lengths of the major and minor axes, respectively, of the fit ellipse. The performance of the correction was validated by Deng et al. [12] through numerical simulations with varying levels of noise and various sphere characteristics. This estimate of the center projection coordinates is used in this study.

\subsection{Tracking the spheres}

Center projection coordinates are determined for all spheres $M$ at all rotation positions $N$. The output of the image analysis step is therefore a set of $\mathrm{M} \times \mathrm{N}$ observed pixel column and row coordinates $\left(u_{\mathrm{obs}}, v_{\mathrm{obs}}\right)$. Given the size of the dataset, a certain level of automation is needed to ensure the continued sanity of the user. A sphere projection tracking procedure is implemented to reduce user input. For each sphere, center projection coordinate gradients from radiograph $n-2$ to radiograph $n-1$ are used to predict the image location of the projected sphere in radiograph $n$. Furthermore, the size of the projected sphere at radiograph $n-1$ is used to generate an appropriately-sized crop region for center projection estimation.

\subsection{Projected sphere overlaps}

Overlaps in the projected spheres introduce errors in the estimation of center projections. For the purpose of simplicity, and due to the fact that overlaps only account for a few percent of all data points, those data points that correspond to sphere overlaps are not considered in the objective function to be minimized. The sphere tracking algorithm is adapted to skip over these overlaps. For each sphere, the radiographs in which the sphere has overlaps are determined by manually scanning the set of radiographs. Overlap 'events' are clusters of sequential radiographs for which a sphere exhibits overlap. The projected sphere position after an overlap event is determined by multiplying the number of radiographs in the specific event $\Delta n$ by the last image gradients prior to the event. The data points corresponding to overlap events are not included in the minimization procedure.

\subsection{Error in observed center projection coordinates}

The pixel coordinates assigned to each center projection deviate from the actual center projection position as a result of errors introduced by image blur, noise, and the image processing step. Furthermore, the presence of rotation stage error motions will also contribute to deviations of the projected sphere centers from the elliptical trajectories they would perform on the detector space under ideal rotation. To evaluate the magnitude of these errors, the set of observed center projection coordinates $\left\{u_{\mathrm{obs}}(m, n), v_{\mathrm{obs}}(m, n)\right\}$ is compared to the exact center projection coordinates $\left\{u_{\text {exact }}(m, n), v_{\text {exact }}(m, n)\right\}$, which are calculated by performing forward projection on the known geometrical parameters of the simulation and under ideal rotation.

The errors in the estimated center projection coordinates $\left\{\delta u_{\text {error }}(m, n), \delta v_{\text {error }}(m, n)\right\}$ are plotted in histogram form for simulation 1 in figure 8 . As a measure of dispersion, the $2.5 \%$ and $97.5 \%$ quantiles (corresponding to a $95 \%$ coverage interval centered at the median error value) are indicated by dashed vertical lines and their values are shown adjacent to the lines. The shape and magnitudes of error distributions were similar for all simulated data sets. The 
$2.5 \%$ and $97.5 \%$ quantiles for all simulations are shown in table 6. Center projection errors were consistently within 0.3 pixels for all projected spheres in all radiographs.

Table 6. Lower and upper boundaries for $95 \%$ of observed center projection errors designated by the $2.5 \%$ and $97.5 \%$ quantiles, respectively. All values are in pixels.

\begin{tabular}{c|cc|cc}
$\boldsymbol{s}$ & \multicolumn{2}{|c|}{$\mathbf{U}$ coordinate quantiles } & \multicolumn{2}{c}{ V coordinate quantiles } \\
& $\mathbf{2 . 5} \%$ & $\mathbf{9 7 . 5} \%$ & $\mathbf{2 . 5} \%$ & $\mathbf{9 7 . 5} \%$ \\
\hline 1 & -0.1104 & 0.1167 & -0.1051 & 0.1046 \\
2 & -0.1090 & 0.1166 & -0.1014 & 0.1047 \\
3 & -0.1162 & 0.1095 & -0.1027 & 0.1050 \\
4 & -0.1070 & 0.1148 & -0.1031 & 0.1033 \\
5 & -0.1089 & 0.1136 & -0.1029 & 0.1039 \\
6 & -0.1176 & 0.1063 & -0.1048 & 0.1033 \\
7 & -0.1081 & 0.1195 & -0.1039 & 0.1034 \\
8 & -0.1092 & 0.1171 & -0.1029 & 0.1046 \\
9 & -0.1176 & 0.1117 & -0.1054 & 0.1032 \\
10 & -0.1145 & 0.1041 & -0.1025 & 0.1050
\end{tabular}

\section{Measurement of the simulated geometry}

\subsection{Convergence testing}

The measurement procedure was tested for convergence to the global minimum. Convergence testing comprises executing the minimization procedure using the exact (known) values of the input quantities, i.e. sphere center coordinates and center projection coordinates, while varying the initial values of the solvable parameters. The minimization for convergence testing is repeated 1000 times, each time randomly varying the initial values of solvable parameters from corresponding uniform distributions about a central value (table 7) and ensuring that the solver search region (set as upper and lower solver search boundaries for each parameter) contains the corresponding simulated value. The concept of convergence testing is illustrated in figure 9. Errors in solved parameters were observed to be larger when the true values were close to the corresponding search boundaries set in the Global Search function. It is therefore important to ensure that the search boundaries are large enough to avoid this shortfall.

Convergence testing was performed for all simulated data sets. Errors between the solved geometrical parameter values and the actual simulated values for simulation 1 are shown in histogram form in figures 10 and 11 for instrument geometrical parameters and in figures 12 and 13 for reference object geometrical parameters. Since the distributions of solved parameters were not normal in shape, the $2.5 \%$ and $97.5 \%$ quantiles corresponding to the 95 $\%$ spread of the respective result are denoted by the solid vertical lines [13]; their values are shown next to the solid lines. The simulated value is denoted by the dotted line, corresponding to an error of zero. Dashed lines correspond to the mean of the solved values. Some distributions were not symmetrical about the true values, which could be an indication of parameter coupling. Results for all simulated datasets are presented in figures 14 and 15, 
where the mean error is given by the circular marker and $95 \%$ of the data points are contained within the associated error bars.

Table 7. Variation of initial values of solvable parameters for convergence testing.

\begin{tabular}{c|c|c|c} 
Parameter & Central value & Variation & Distribution \\
\hline$x_{\mathrm{D}}$ & 0 & $\pm 10 \mathrm{~mm}$ & Uniform \\
$y_{\mathrm{D}}$ & 0 & $\pm 10 \mathrm{~mm}$ & Uniform \\
$z_{\mathrm{D}}$ & -1177 & $\pm 10 \mathrm{~mm}$ & Uniform \\
$\rho_{\mathrm{X}}$ & 0 & \pm 10 degrees & Uniform \\
$\rho_{\mathrm{Y}}$ & 0 & \pm 10 degrees & Uniform \\
$\rho_{\mathrm{Z}}$ & 0 & \pm 10 degrees & Uniform \\
$x_{\mathrm{P}}$ & 0 & $\pm 10 \mathrm{~mm}$ & Uniform \\
$y_{\mathrm{P}}$ & 0 & $\pm 10 \mathrm{~mm}$ & Uniform \\
$z_{\mathrm{P}}$ & -400 & $\pm 10 \mathrm{~mm}$ & Uniform \\
$z_{\mathrm{R}}$ & -400 & $\pm 10 \mathrm{~mm}$ & Uniform \\
$\theta$ & 0 & \pm 10 degrees & Uniform \\
$\varphi$ & 0 & \pm 10 degrees & Uniform \\
$\eta$ & 0 & \pm 10 degrees & Uniform
\end{tabular}

Convergence testing allows us to identify parameter coupling, which could influence parameter identifiability [14]. For example, the errors in solved Z positions of reference object, rotation axis, and detector are consistently offset in the same direction, i.e. closer to the source, and the magnitude of error in $z_{\mathrm{D}}$ is approximately the error in both $z_{\mathrm{R}}$ and $z_{\mathrm{P}}$ multiplied by the magnification factor of 2.94. Convergence results indicate that, even in the presence of exact input quantities, the minimization procedure has intrinsic limitations in its ability to solve the geometrical parameters.

\subsection{Geometrical measurement results}

The input quantities for the minimization procedure were the nominal (unperturbed) sphere center coordinates in the local object frame and the observed center projection coordinates $\left(u_{\mathrm{obs}}, v_{\mathrm{obs}}\right)$. Initial parameter values are set to their central values in Table 7 . Errors between solved parameter values and simulated values are shown for instrument geometrical parameters in figure 16 and for reference object geometrical parameters figure 17. The solved values and corresponding errors are presented together with the simulated values in tables A1-A5 in Appendix A. Errors in solved detector Z position were within $105 \mu \mathrm{m}$ for all simulations and errors in rotation axis and reference object $Z$ positions were within approximately $35 \mu \mathrm{m}$. $X$ and $Y$ positions of detector and reference object were solved to within $5 \mu \mathrm{m}$ from their simulated values. Detector out-of-plane rotations $\theta$ and $\varphi$ solved to within 10 arcseconds and 3 arcseconds, respectively, while detector in-plane rotation $\eta$ consistently solved to within 1 arcsecond. Coupling between the $\mathrm{Z}$ positions of rotation stage, reference object, and detector is evident.

Errors between observed center projection coordinates and minimized center projection coordinates, i.e. modelled after minimization, and the observed center projection coordinates 
are analyzed. Histograms for these center projection coordinate errors from simulation 1 are shown in figure $18.95 \%$ of the data is indicated by dashed vertical lines corresponding to the $2.5 \%$ and $97.5 \%$ quantiles, respectively; their values are shown adjacent to the lines. The shape and magnitudes of error distributions were similar for all simulated data sets. The 2.5 $\%$ and $97.5 \%$ quantiles for all simulations are shown in table 8. Comparison of the errors between minimized and observed center projection coordinates to the errors between exact and observed center projection coordinates from table 6 and figure 8 indicates that the two data sets are very similar.

Table 8. Lower and upper boundaries for $95 \%$ of errors between observed and minimized center projection coordinates, designated by the $2.5 \%$ and $97.5 \%$ quantiles, respectively. All values are in pixels.

\begin{tabular}{c|cc|cc} 
& \multicolumn{2}{|c|}{ U coordinate quantiles } & \multicolumn{2}{c}{ V coordinate quantiles } \\
& $\mathbf{2 . 5} \%$ & $\mathbf{9 7 . 5} \%$ & $\mathbf{2 . 5} \%$ & $\mathbf{9 7 . 5} \%$ \\
\hline 1 & -0.1095 & 0.1144 & -0.1039 & 0.1063 \\
2 & -0.1094 & 0.1090 & -0.1046 & 0.1032 \\
3 & -0.1131 & 0.1118 & -0.1040 & 0.1065 \\
4 & -0.1119 & 0.1096 & -0.1033 & 0.1048 \\
5 & -0.1125 & 0.1095 & -0.1055 & 0.1029 \\
6 & -0.1105 & 0.1111 & -0.1059 & 0.1050 \\
7 & -0.1112 & 0.1117 & -0.1027 & 0.1043 \\
8 & -0.1094 & 0.1121 & -0.1025 & 0.1054 \\
9 & -0.1097 & 0.1113 & -0.1038 & 0.1053 \\
10 & -0.1092 & 0.1088 & -0.1055 & 0.1030
\end{tabular}

\subsection{Instrument adjustment}

The output from the proposed geometrical measurement procedure is used to correct for instrument misalignments in the simulated CT measurement of a separate test object. The test object consists of a ball plate with 15 spheres of $2 \mathrm{~mm}$ diameter; 14 of these spheres form two crossing diagonal lines and one sphere is at a horizontal extremity of the plate (figure 19). For simplicity, only the spheres are simulated. The areal extent of the spheres is approximately $105 \mathrm{~mm} \times 105 \mathrm{~mm}$.

Some experimental CT instruments allow the detector position and orientation to be controlled by kinematic axes or by re-mounting of the detector. In these instruments, detector lateral position $x_{\mathrm{D}}, y_{\mathrm{D}}$ and detector orientation $\theta, \varphi, \eta$ can therefore be adjusted to zero using their measured values. SRD and SDD in the acquisition metafile (typically a text file containing relevant acquisition parameters used in tomographic reconstruction) are then adapted to the measured rotation axis position $z_{\mathrm{R}}$ and detector longitudinal position $z_{\mathrm{D}}$, respectively; the voxel size in the metafile is appropriately adjusted to reflect the new SRD and SDD.

Differences between the set of solved and actual instrument geometrical parameters result in residual misalignments after correction. We evaluate the performance of correcting the 
instrument geometry from the set of measured geometrical parameters as follows. Radiographs of the test object are simulated under aligned instrument geometry, under 10 misaligned instrument geometries and under 10 adjusted instrument geometries, i.e. corrected for detector lateral position and orientation. Instrument geometrical parameters for aligned and misaligned acquisitions are provided in table 3, while the same parameters for the adjusted acquisitions are shown in table 9. Note: Scorpius $\mathrm{XLab}^{\circledR}$ rounds linear positions (in $\mathrm{mm}$ ) to 3 decimal places and angular positions (in degrees) to four decimal places.

Tomographic reconstruction from the simulated radiographs is performed on CT Pro 3D (Nikon Metrology, UK). Reconstruction of the aligned and misaligned datasets is performed assuming aligned reconstruction geometry. Discrepancies in SRD and SDD between aligned reconstruction geometry and misaligned acquisition geometry can be partially corrected by implementing voxel rescaling per the method in [15]. Voxel scaling factors for each misaligned acquisition of the ball plate are determined from center-to-center distance (C2C) measurements on the same misaligned acquisition of the $\mathrm{CT}^{2}$ and applied to the respective reconstructed volume of the test object. Reconstruction of the adjusted datasets is performed under aligned detector lateral position and orientation. The values for SRD, SDD, and voxel size in the adjusted acquisition metafile for CT Pro 3D (.xtekct) are set to correspond to the measured $z_{\mathrm{R}}$ and $z_{\mathrm{D}}$ values from tables A1 and A3, respectively, in Appendix A.

Dimensional measurements are performed on the reconstructed misaligned datasets without and with voxel scaling, henceforth 'misaligned' and 'rescaled', and on the adjusted datasets. Results are compared to equivalent measurements from aligned acquisition. Volumetric grey value models are imported into VGStudio MAX 3.0 (Volume Graphics, GmbH). Surfaces are generated from the volumetric models by applying 'advanced' (local) surface determination to automatically evaluated initial grey value thresholds. The search distance for advanced surface determination is set to the default 4 voxels. The resulting surface model is converted to a three-dimensional coordinate point cloud by sampling at intervals of 1 voxel in all coordinate directions.

Point clouds are then processed in MATLAB. First, the point cloud is segmented such that the coordinate points corresponding to the surface of each sphere are separated from surface points of other spheres. Subsequently, spheres are least-squared fit [16] to each segmented set of coordinate points. Fit sphere centers are used to determine $\mathrm{C} 2 \mathrm{C}$ between all combinations of spheres. Errors of misaligned, rescaled, and adjusted $\mathrm{C} 2 \mathrm{C}$ relative to the same measurements from the aligned acquisition are plotted in figure 20. Results from all simulations are shown together. $\mathrm{C} 2 \mathrm{C}$ errors in the misaligned dataset were as large as 1175 $\mu \mathrm{m}$. Voxel rescaling reduced these errors to below $220 \mu \mathrm{m}$, while errors after adjustment were within $2 \mu \mathrm{m}$ of the aligned acquisition values.

Errors in sphere fit radii relative to aligned acquisition radii are shown in figure 21. Radius errors were mostly unchanged for misaligned and rescaled datasets; in both cases, the largest radius errors were approximately $100 \mu \mathrm{m}$. After adjustment, radius errors were within $1 \mu \mathrm{m}$. Observed errors after adjustment are relatively small when compared to the effects of other error sources in the CT measurement procedure. These results therefore suggest that the geometrical measurement procedure provides a robust estimation of the instrument geometry. 
Table 9. Instrument parameters for adjusted geometry acquisitions. The values in this table are rounded to 3 decimal places for linear positions in $\mathrm{mm}$ and 4 decimal places for angular positions in degrees to correspond to rounding of geometrical parameters in Scorpius $\mathrm{XLab}^{\circledR}$.

\begin{tabular}{c|ccccccc}
$\begin{array}{c}\text { Adjusted } \\
\text { acquisition }\end{array}$ & $\begin{array}{c}\boldsymbol{x}_{\mathbf{D}} \\
/ \mathbf{m m}\end{array}$ & $\begin{array}{c}\boldsymbol{y}_{\mathbf{D}} \\
/ \mathbf{m m}\end{array}$ & $\begin{array}{c}\mathbf{z}_{\mathbf{D}} \\
/ \boldsymbol{\mu} \mathbf{m}\end{array}$ & $\begin{array}{c}\mathbf{Z}_{\mathbf{R}} \\
/ \mathbf{m m}\end{array}$ & $\begin{array}{c}\boldsymbol{\theta} \\
/ \mathbf{0}\end{array}$ & $\begin{array}{c}\boldsymbol{\varphi} \\
/ \mathbf{0}\end{array}$ & $\boldsymbol{\eta}$ \\
\hline 1 & -0.001 & 0.000 & -1175.443 & -402.545 & -0.0027 & -0.0002 & 0.0003 \\
2 & -0.001 & -0.004 & -1181.643 & -402.676 & -0.0010 & -0.0008 & 0.0001 \\
3 & 0.001 & 0.003 & -1173.509 & -399.815 & 0.0007 & -0.0007 & -0.0001 \\
4 & -0.001 & 0.004 & -1172.660 & -398.325 & -0.0007 & 0.0002 & 0.0001 \\
5 & 0.000 & 0.004 & -1175.213 & -397.396 & 0.0001 & -0.0004 & 0.0000 \\
6 & 0.001 & 0.002 & -1174.423 & -402.221 & -0.0001 & 0.0003 & -0.0002 \\
7 & -0.001 & -0.003 & -1174.569 & -399.587 & -0.0020 & -0.0006 & 0.0002 \\
8 & -0.001 & -0.003 & -1178.078 & -400.184 & -0.0009 & -0.0002 & 0.0002 \\
9 & 0.000 & -0.004 & -1175.446 & -402.929 & 0.0001 & -0.0005 & 0.0000 \\
10 & 0.001 & 0.003 & -1180.288 & -400.977 & 0.0003 & -0.0003 & -0.0002
\end{tabular}

\section{Conclusion and discussion}

In this simulation study, we evaluate the performance of a procedure to measure the CT instrument geometry by minimization of reprojection errors from radiographs acquired of the $\mathrm{CT}^{2}$ reference object. The proposed geometrical measurement procedure can be applied with other reference objects. Realistic effects - such as rotation stage error motions, uncertainty in the reference object sphere center coordinates, and image blur and noise - were included in this simulation study to approximate the errors expected in experimental implementation. The proposed procedure is applied to measure 10 simulated acquisition geometries; the solved geometrical parameters are compared to the 'true' simulated parameters. Errors in solved parameters are relatively small. Detector $X$ and $Y$ positions are solved to within $5 \mu \mathrm{m}$ of the true value, while detector $\mathrm{Z}$ position is solved to within $105 \mu \mathrm{m}$. Errors in detector outof-plane rotations $\theta$ and $\varphi$ are within 10 arc seconds and 3 arc seconds, respectively, while errors in detector in-plane rotation $\eta$ are within 1 arc second. The $\mathrm{Z}$ position of the axis of rotation is solved to within $35 \mu \mathrm{m}$ of the true value. The results from convergence testing (see section 5.1) indicate that coupling between $Z$ positions of the rotation axis $z_{R}$ and detector $z_{D}$ resulted in systematic offsets of their solved values. The solved geometrical parameters from the proposed measurement procedure can be used to correct the CT instrument, either by physical adjustment of the components or by software correction. Errors in the solved geometrical parameters will result in residual geometrical errors of the corrected instrument. The CT measurement of a separate ball plate is simulated under aligned, misaligned (without and with voxel rescaling), and adjusted instrument geometries for each of the 10 simulated datasets. Dimensional measurements on the reconstructed datasets are compared. Center-tocenter distance measurement errors were as large as $1175 \mu \mathrm{m}$ in the misaligned dataset without voxel rescaling. These errors were reduced to a maximum of $220 \mu \mathrm{m}$ after voxel rescaling and to within $2 \mu \mathrm{m}$ after adjustment. Radius errors as large as $100 \mu \mathrm{m}$ on $2 \mathrm{~mm}$ spheres in misaligned datasets were reduced to within $1 \mu \mathrm{m}$ after adjustment. Errors in the 
reconstructed volume in the presence of residual geometrical misalignments are therefore relatively small, thereby validating the robustness of the geometrical measurement procedure.

Calibration is defined in the VIM [17] as the (a) comparison to a traceable reference and (b) assessment of uncertainty in the comparison. Thus far, calibration of CT geometrical parameters as per the metrological definition has not been demonstrated. The use of reference objects is common in previously proposed estimation methods. However, the application of a reference object with one or more traceable dimensional features to the measurement of the CT geometry has not been shown. Uncertainty in previous literature on estimation of geometrical parameters is solely calculated from errors in the observed center projection; other sources of error, such as uncertainty in the traceable reference features, are not considered [3]. Assessment of uncertainty for quantities estimated by minimization does not lend itself to analytical methods, such as the method described in the GUM [1]. While we demonstrate measurement of the CT geometrical parameters by comparison to a traceable reference in this study, a method to assess uncertainty in the solved parameter values is not yet available and is a topic of further work in our research group.

\section{Acknowledgments}

The research presented here was made possible by funding from the European Union H2020 FlexCT project (grant agreement 752672), from the European Union FP7 INTERAQCT project (grant agreement 607817), and from the Belgian American Educational Foundation's Fellowship for Graduate Research in Belgium. 


\section{Appendix A}

Table A1. Comparison of solved detector position after minimization of reprojection errors to true simulated values for each simulation $s$. True and solved values are in $\mathrm{mm}$, while errors are in $\mu \mathrm{m}$.

\begin{tabular}{|c|c|c|c|c|c|c|c|c|c|}
\hline \multirow[b]{2}{*}{$S$} & \multicolumn{3}{|c|}{$x_{\mathbf{D}}$} & \multicolumn{3}{|c|}{$y_{D}$} & \multicolumn{3}{|c|}{$z_{D}$} \\
\hline & $\begin{array}{l}\text { True } \\
/ \mathrm{mm}\end{array}$ & $\begin{array}{c}\text { Solved } \\
/ \mathrm{mm}\end{array}$ & $\begin{array}{l}\text { Error } \\
/ \mu \mathrm{m}\end{array}$ & $\begin{array}{l}\text { True } \\
/ \mathrm{mm}\end{array}$ & $\begin{array}{l}\text { Solved } \\
/ \mathrm{mm}\end{array}$ & $\begin{array}{l}\text { Error } \\
/ \mu \mathrm{m}\end{array}$ & $\begin{array}{l}\text { True } \\
/ \mathrm{mm}\end{array}$ & $\begin{array}{l}\text { Solved } \\
/ \mathrm{mm}\end{array}$ & $\begin{array}{c}\text { Error } \\
/ \mu \mathrm{m}\end{array}$ \\
\hline 1 & 1.2590 & 1.2600 & 1.0427 & -1.3700 & -1.3699 & 0.0739 & -1175.4430 & -1175.3516 & 91.4167 \\
\hline 2 & 1.6230 & 1.6238 & 0.7558 & 1.8820 & 1.8856 & 3.6289 & -1181.6430 & -1181.5410 & 102.0365 \\
\hline 3 & -1.4920 & -1.4926 & -0.6351 & 1.8290 & 1.8257 & -3.2696 & -1173.5090 & -1173.4267 & 82.3333 \\
\hline 4 & 1.6540 & 1.6547 & 0.6736 & -0.0590 & -0.0631 & -4.0903 & 172.6600 & -1172.5970 & 63.0269 \\
\hline 5 & 0.5290 & 0.5295 & 0.4689 & 1.2010 & 1.1974 & -3.6089 & -1175.2130 & -1175.1348 & 78.2149 \\
\hline 6 & -1.6100 & -1.6110 & -1.0414 & -1.4330 & -1.4354 & -2.3963 & -1174.4230 & -1174.3373 & 85.6979 \\
\hline 7 & -0.8860 & -0.8848 & 1.2391 & -0.3130 & -0.3099 & 3.0915 & -1174.5690 & -1174.4803 & 88.6874 \\
\hline 8 & 0.1880 & 0.1891 & 1.0990 & 1.6630 & 1.6664 & 3.4178 & -1178.0780 & -1177.9785 & 99.4698 \\
\hline 9 & 1.8300 & 1.8296 & -0.3719 & 1.1690 & 1.1730 & 3.9766 & -1175.4460 & -1175.3467 & 99.3094 \\
\hline 10 & 1.8600 & 1.8591 & -0.9451 & 1.8380 & 1.8346 & -3.3946 & -1180.2880 & -1180.2002 & 87.8361 \\
\hline
\end{tabular}


Table A2. Comparison of solved detector orientation after minimization of reprojection errors to true simulated values for each simulation. True and solved values are in degrees $\left({ }^{\circ}\right)$, while errors are in arcseconds $\left(1 / 3600^{\circ}\right)$.

\begin{tabular}{|c|c|c|c|c|c|c|c|c|c|}
\hline \multirow[b]{2}{*}{$S$} & \multicolumn{3}{|c|}{$\boldsymbol{\theta}$} & \multicolumn{3}{|c|}{$\varphi$} & \multicolumn{3}{|c|}{$\eta$} \\
\hline & $\begin{array}{c}\text { True } \\
10\end{array}$ & $\begin{array}{c}\text { Solved } \\
/{ }^{\circ}\end{array}$ & $\begin{array}{c}\text { Error } \\
\text { /arcsecs }\end{array}$ & $\begin{array}{c}\text { True } \\
/ 0\end{array}$ & $\begin{array}{c}\text { Solved } \\
10^{\circ}\end{array}$ & $\begin{array}{c}\text { Error } \\
\text { /arcsecs }\end{array}$ & $\begin{array}{c}\text { True } \\
/{ }^{\circ}\end{array}$ & $\begin{array}{c}\text { Solved } \\
1^{\circ}\end{array}$ & $\begin{array}{c}\text { Error } \\
\text { /arcsecs }\end{array}$ \\
\hline 1 & -0.6756 & -0.6729 & 9.8741 & -0.0989 & -0.0987 & 0.5852 & -0.7867 & -0.7870 & -0.9400 \\
\hline 2 & 0.5886 & 0.5896 & 3.4445 & -0.8324 & -0.8316 & 2.9650 & 0.9238 & 0.9237 & -0.4427 \\
\hline 3 & -0.3776 & -0.3783 & -2.5576 & -0.5420 & -0.5413 & 2.6222 & -0.9907 & -0.9906 & 0.4905 \\
\hline 4 & 0.0571 & 0.0578 & 2.5552 & 0.8267 & 0.8265 & -0.6820 & 0.5498 & 0.5497 & -0.3554 \\
\hline 5 & -0.6687 & -0.6688 & -0.3608 & -0.6952 & -0.6948 & 1.4780 & 0.6346 & 0.6346 & -0.1787 \\
\hline 6 & 0.2040 & 0.2041 & 0.4834 & 0.6516 & 0.6513 & -1.0173 & 0.7374 & 0.7376 & 0.5548 \\
\hline 7 & -0.4741 & -0.4721 & 7.1076 & 0.0767 & 0.0773 & 2.0130 & -0.8311 & -0.8313 & -0.8109 \\
\hline 8 & 0.3082 & 0.3091 & 3.3472 & 0.9923 & 0.9925 & 0.5815 & -0.2004 & -0.2006 & -0.5417 \\
\hline 9 & 0.3784 & 0.3783 & -0.4235 & -0.8436 & -0.8431 & 1.7183 & -0.4803 & -0.4803 & -0.0220 \\
\hline 10 & 0.4963 & 0.4960 & -0.9362 & -0.1146 & -0.1143 & 1.1016 & 0.6001 & 0.6003 & 0.6440 \\
\hline
\end{tabular}


Table A310. Comparison of solved rotation axis position after minimization of reprojection errors to true simulated values for each simulation. True and solved values are in $\mathrm{mm}$, while errors are in $\mu \mathrm{m}$.

\begin{tabular}{c|ccc}
$\boldsymbol{s}$ & $\begin{array}{c}\mid c \\
\mathbf{Z}_{\mathbf{R}} \\
\text { True } \\
/ \mathbf{m m}\end{array}$ & $\begin{array}{c}\text { Solved } \\
/ \mathbf{m m}\end{array}$ & $\begin{array}{c}\text { Error } \\
/ \boldsymbol{\mu m}\end{array}$ \\
\hline 1 & -402.5450 & -402.5133 & 31.7315 \\
2 & -402.6760 & -402.6415 & 34.4909 \\
3 & -399.8150 & -399.7863 & 28.7070 \\
4 & -398.3250 & -398.3028 & 22.2407 \\
5 & -397.3960 & -397.3696 & 26.4214 \\
6 & -402.2210 & -402.1912 & 29.8046 \\
7 & -399.5870 & -399.5573 & 29.6724 \\
8 & -400.1840 & -400.1508 & 33.1642 \\
9 & -402.9290 & -402.8952 & 33.8073 \\
10 & -400.9770 & -400.9468 & 30.2234
\end{tabular}


Table A4. Comparison of solved reference object position after minimization of reprojection errors to true simulated values for each simulation. True and solved values are in $\mathrm{mm}$, while errors are in $\mu \mathrm{m}$.

\begin{tabular}{|c|c|c|c|c|c|c|c|c|c|}
\hline \multirow[b]{2}{*}{$s$} & \multicolumn{3}{|c|}{$x_{\mathbf{P}}$} & \multicolumn{3}{|c|}{$y_{P}$} & \multicolumn{3}{|c|}{$z_{P}$} \\
\hline & $\begin{array}{l}\text { True } \\
/ \mathrm{mm}\end{array}$ & $\begin{array}{l}\text { Solved } \\
/ \mathrm{mm}\end{array}$ & $\begin{array}{l}\text { Error } \\
/ \mu \mathrm{m}\end{array}$ & $\begin{array}{l}\text { True } \\
/ \mathrm{mm}\end{array}$ & $\begin{array}{c}\text { Solved } \\
/ \mathrm{mm}\end{array}$ & $\begin{array}{l}\text { Error } \\
/ \mu \mathrm{m}\end{array}$ & $\begin{array}{l}\text { True } \\
/ \mathrm{mm}\end{array}$ & $\begin{array}{l}\text { Solved } \\
/ \mathrm{mm}\end{array}$ & $\begin{array}{l}\text { Error } \\
/ \mu \mathrm{m}\end{array}$ \\
\hline 1 & 1.0051 & 1.0061 & 1.0260 & 1.3629 & 1.3632 & 0.2826 & -400.5934 & -400.5614 & 32.0206 \\
\hline 2 & -0.9796 & -0.9791 & 0.4860 & -0.9829 & -0.9815 & 1.3863 & -398.6767 & -398.6411 & 35.6076 \\
\hline 3 & 0.0238 & 0.0233 & -0.5026 & 1.2571 & 1.2562 & -0.8926 & -399.6589 & -399.6310 & 27.8658 \\
\hline 4 & 0.7963 & 0.7966 & 0.3135 & -1.0259 & -1.0273 & -1.4259 & -399.8011 & -399.7797 & 21.3906 \\
\hline 5 & 1.5636 & 1.5638 & 0.2218 & 1.7171 & 1.7160 & -1.0979 & -398.3312 & -398.3059 & 25.2578 \\
\hline 6 & 1.8372 & 1.8362 & -1.0487 & -0.6001 & -0.6009 & -0.7714 & -400.8566 & -400.8274 & 29.1669 \\
\hline 7 & 0.1889 & 0.1900 & 1.1174 & -1.2136 & -1.2123 & 1.2633 & -398.9712 & -398.9412 & 30.0043 \\
\hline 8 & -1.4455 & -1.4446 & 0.9163 & -0.9957 & -0.9943 & 1.3517 & -398.9851 & -398.9510 & 34.0834 \\
\hline 9 & -1.4028 & -1.4029 & -0.1128 & 0.4642 & 0.4654 & 1.2131 & -400.4782 & -400.4430 & 35.2490 \\
\hline 10 & -0.9700 & -0.9708 & -0.7862 & -0.1068 & -0.1080 & -1.1630 & -399.7287 & -399.6991 & 29.5716 \\
\hline
\end{tabular}


Table A5. Comparison of solved reference object orientation after minimization of reprojection errors to true simulated values for each simulation. True and solved values are in degrees $\left({ }^{\circ}\right)$, while errors are in arcseconds $\left(1 / 3600^{\circ}\right)$.

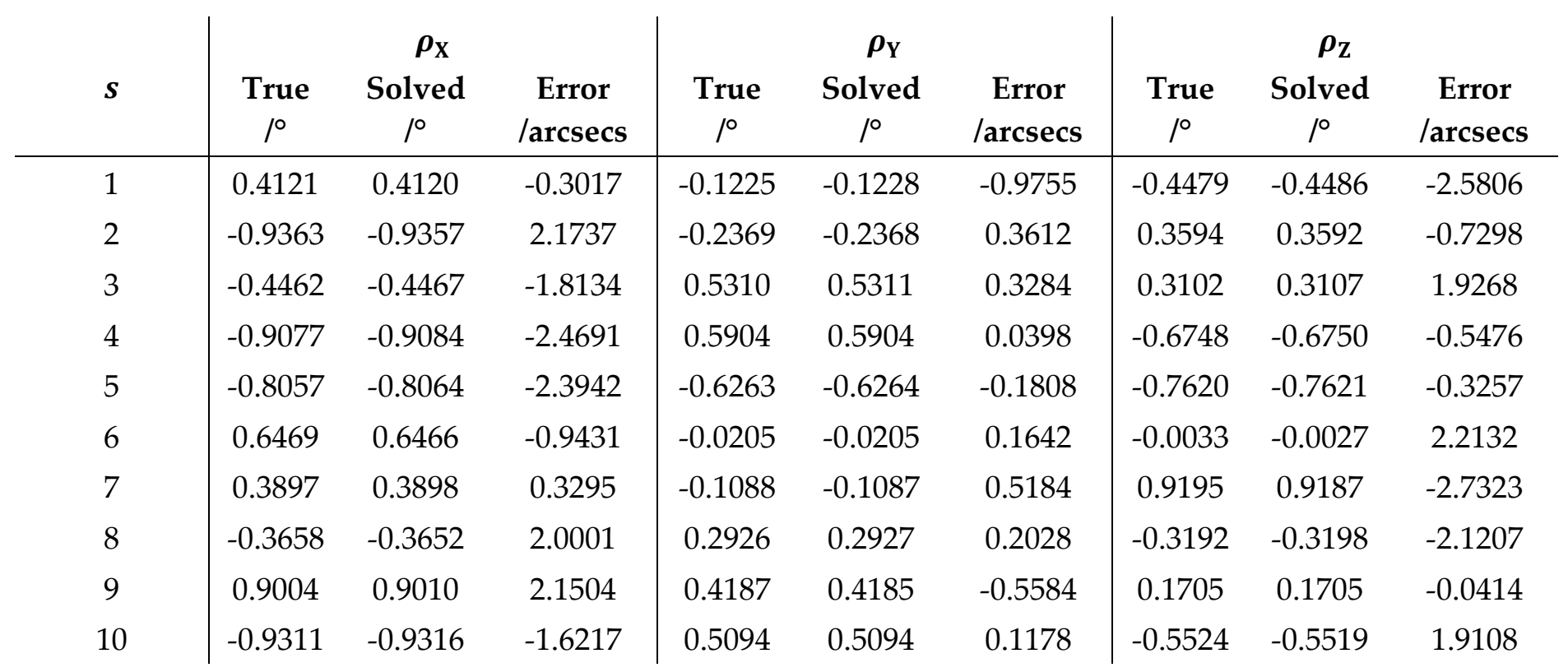




\section{References}

[1] BIPM, IEC, IFCC, ILAC, ISO, IUPAC, et al. JCGM 100 Evaluation of Measurement Data-Guide to the Expression of Uncertainty in Measurement. International Organization for Standardization, 2008.

[2] Ferrucci M. Towards traceability of CT dimensional measurements. In: Carmignato S, Dewulf W, Leach RK, editors. Ind. X-Ray Comput. Tomogr., Berlin, Germany: Springer-Verlag; 2017.

[3] Ferrucci M, Leach RK, Giusca CL, Carmignato S, Dewulf W. Towards geometrical calibration of X-ray computed tomography systems - A review. Meas Sci Technol 2015;26:92003. doi:10.1088/0957-0233/26/9/092003.

[4] Hermanek P, Ferrucci M, Dewulf W, Carmignato S. Optimized reference object for assessment of computed tomography instrument geometry. 7th Conf. Ind. Comput. Tomogr., 2017, p. 7-8.

[5] Hartley R, Zisserman A. Multiple view geometry in computer vision. 2nd ed. Cambridge, UK: Cambridge University Press; 2004. doi:10.1017/CBO9781107415324.004.

[6] Mathworks. Global Search Class 2010.

[7] Mathworks. Multi Start Class 2010.

[8] Cho Y, Moseley DJ, Siewerdsen JH, Jaffray DA. Accurate technique for complete geometric calibration of cone-beam computed tomography systems. Med Phys 2005;32:968-83. doi:10.1118/1.1869652.

[9] Claus BEH. Geometry calibration phantom design for 3D imaging. Proc SPIE 2006;6142:61422E-61422E-12. doi:10.1117/12.652342.

[10] Desbat L, Clackdoyle R, Grezes-Besset L, Mennessier C, Bricault I. Cone-beam imaging of delta functions. IEEE Nucl Sci Symp Conf Rec 2006;5:2859-63. doi:10.1109/NSSMIC.2006.356473.

[11] Clackdoyle R, Mennessier C. Centers and centroids of the cone-beam projection of a ball. Phys Med Biol 2011;56:7371-91. doi:10.1088/0031-9155/56/23/003.

[12] Deng L, Xi X, Li L, Han Y, Yan B. A method to determine the detector locations of the cone-beam projection of the balls ' centers. Phys Med Biol 2015;60:9295-311. doi:10.1088/0031-9155/60/24/9295.

[13] BIPM, IEC, IFCC, ILAC, ISO, IUPAC, et al. JCGM 101: Evaluation of measurement data - Supplement 1 to the "Guide to the expression of uncertainty in measurement" - Propagation of distributions using a Monte Carlo method 2008;JCGM 101:2:90.

[14] Doherty J, Hunt RJ. Two statistics for evaluating parameter identifiability and error reduction. J Hydrol 2009;366:119-27. doi:10.1016/j.jhydrol.2008.12.018.

[15] Stolfi A, De Chiffre L. 3D artefact for concurrent scale calibration in Computed 
Tomography. CIRP Ann - Manuf Technol 2016;65:499-502.

doi:10.1016/j.cirp.2016.04.069.

[16] Hunyadi L. Fitting quadratic curves and surfaces. MATLAB Cent File Exch 2014.

[17] BIPM, IEC, IFCC, ILAC, ISO, IUPAC, et al. JCGM 200 International vocabulary of metrology - Basic and general concepts and associated terms (VIM) 3rd edition (Geneva: International Organization for Standardization) 2012.

\section{Figures}

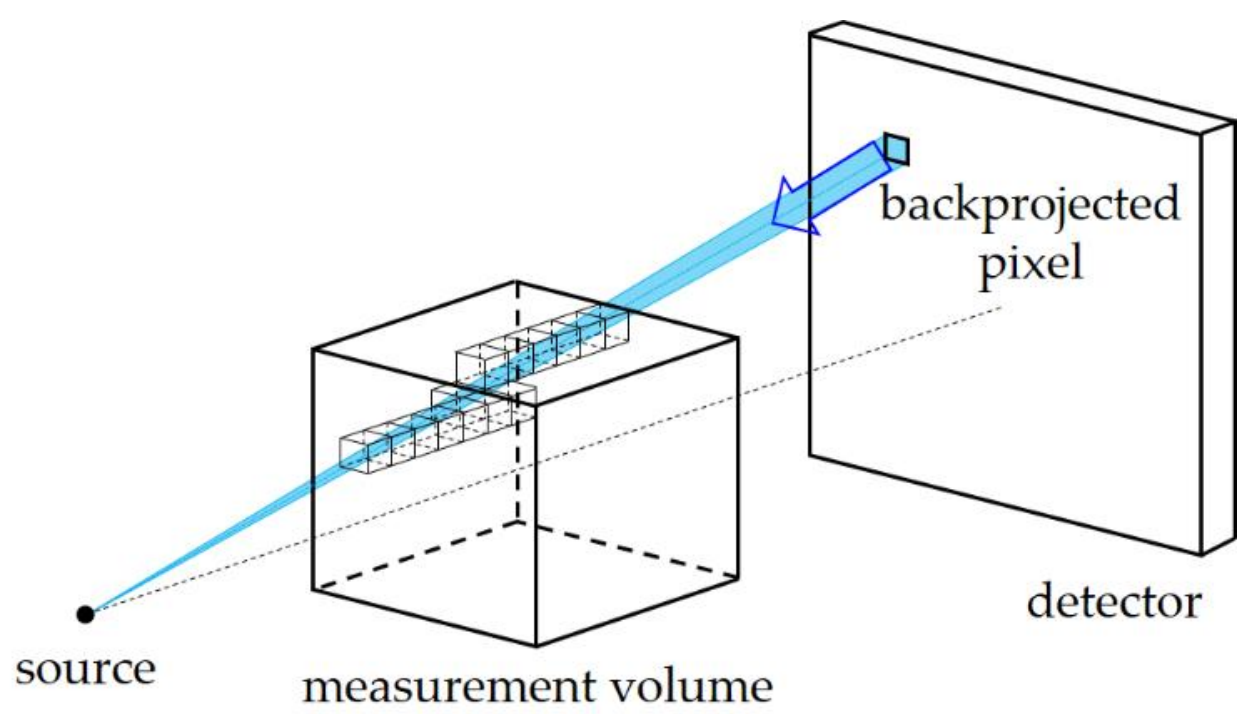

Figure 1. Tomographic reconstruction consists of backprojecting measured X-ray intensities from each pixel through the measurement volume and to the $\mathrm{X}$-ray source focal spot.

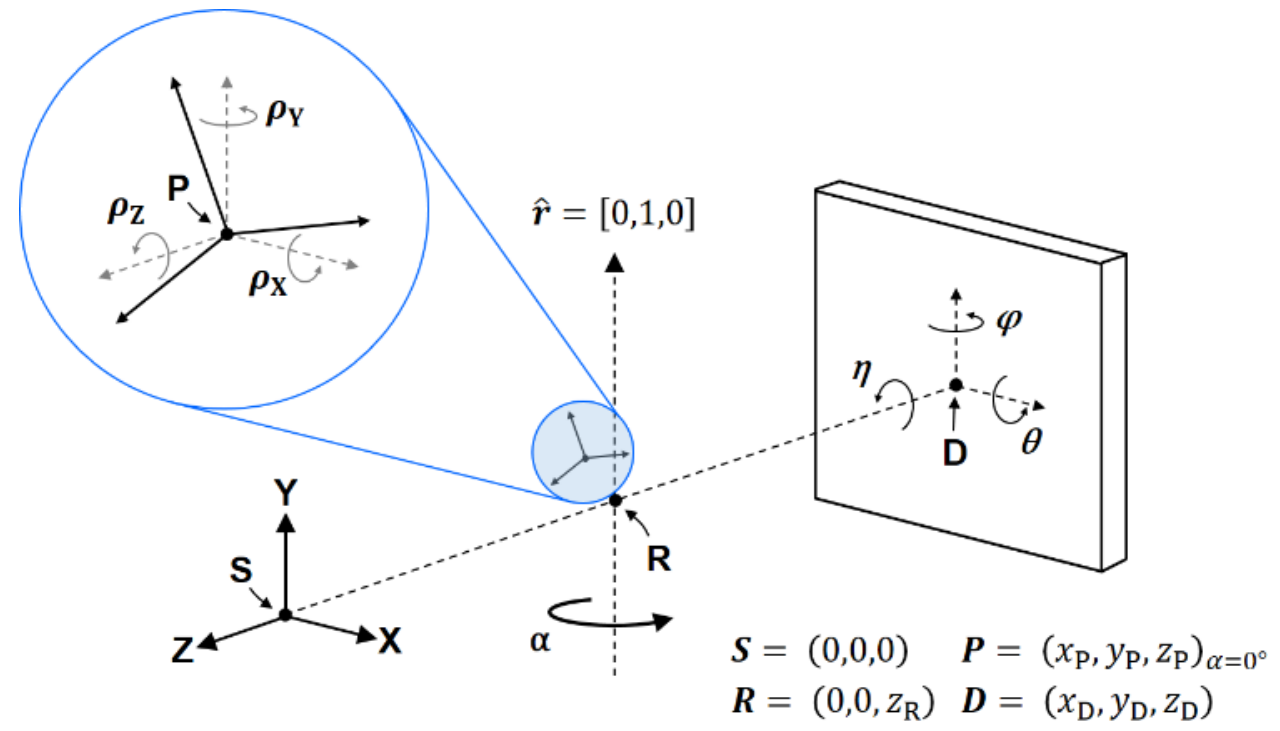

Figure 2. CT geometry with reference object position and orientation. 


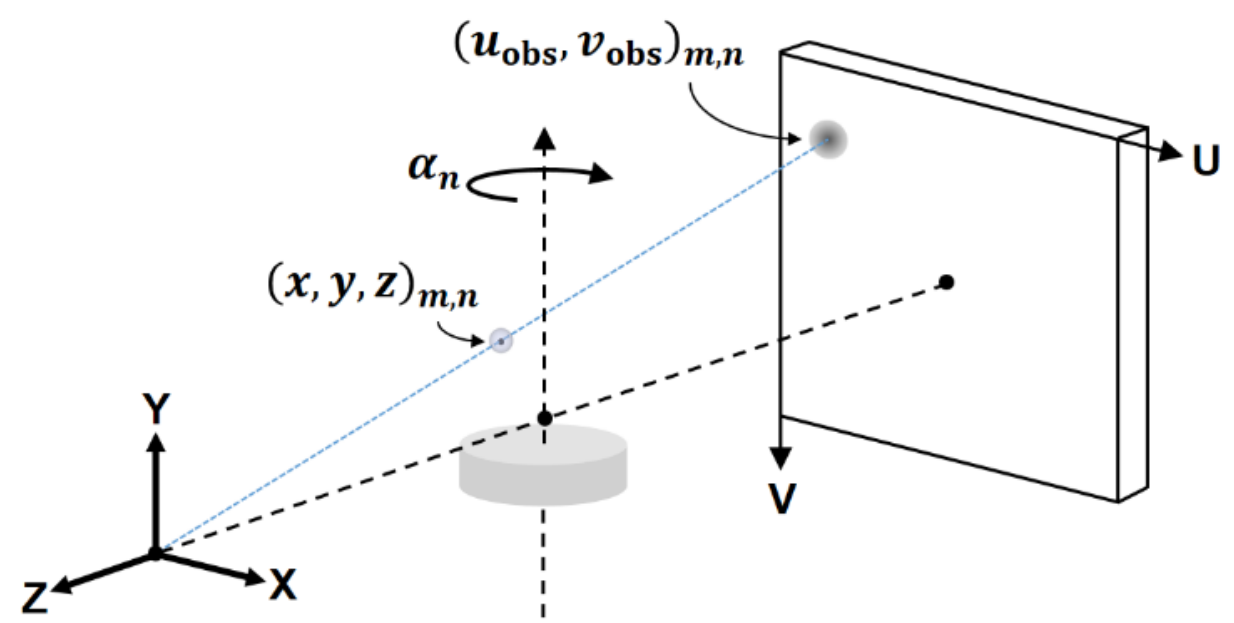

Figure 3. Analysis of each radiograph provides $\mathrm{M}$ center projection coordinates. A complete data acquisition of $\mathrm{N}$ radiographs and subsequent analysis provide a set of $\mathrm{M} \times$ $\mathrm{N}$ center projection coordinates $\left(u_{\mathrm{obs}}, v_{\mathrm{obs}}\right)_{m, n}$.
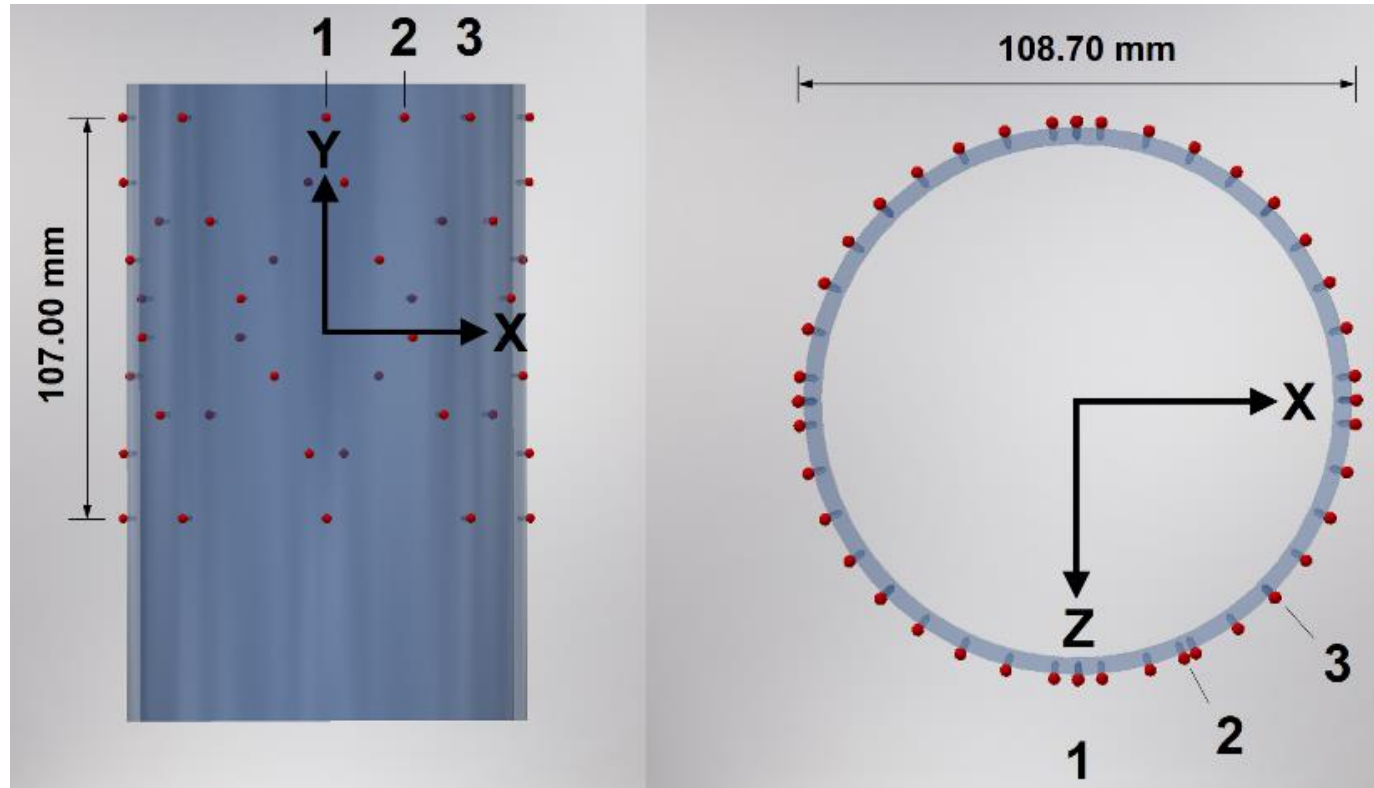

Figure 4. CAD model of the 49-sphere $\mathrm{CT}^{2}$ reference object with local coordinate frame. Left: Side view. Right: Top-down view. Spheres 1, 2, and 3 are indicated for orientation. 


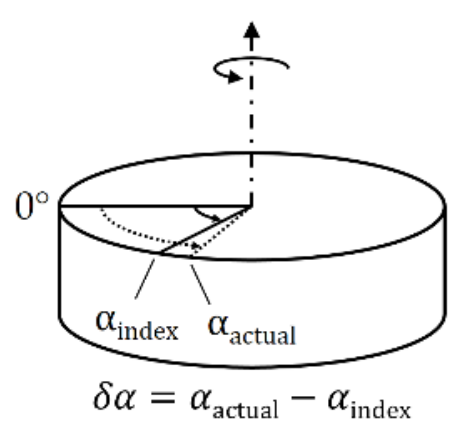

angular indexing error

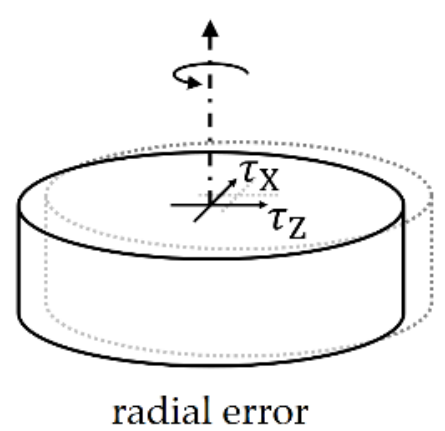

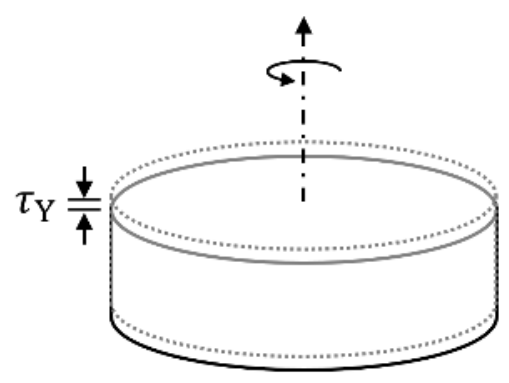

axial error

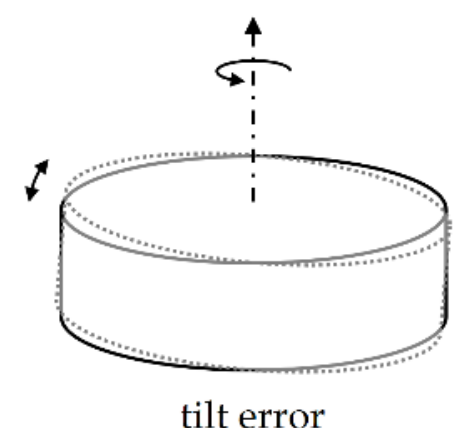

Figure 5. Rotation stage error motions.

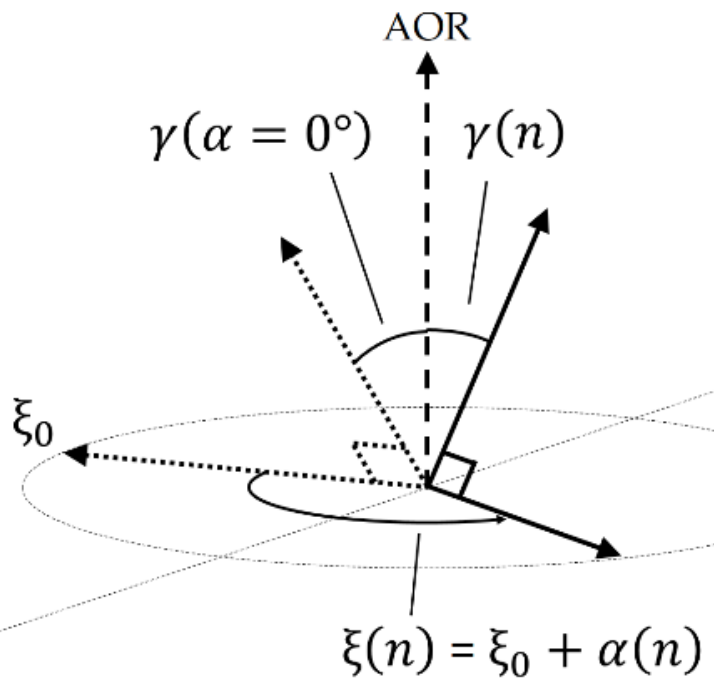

Figure 6. Rotation stage tilt error motion is parameterized by two angles, which vary as a function of rotation position $n$. 


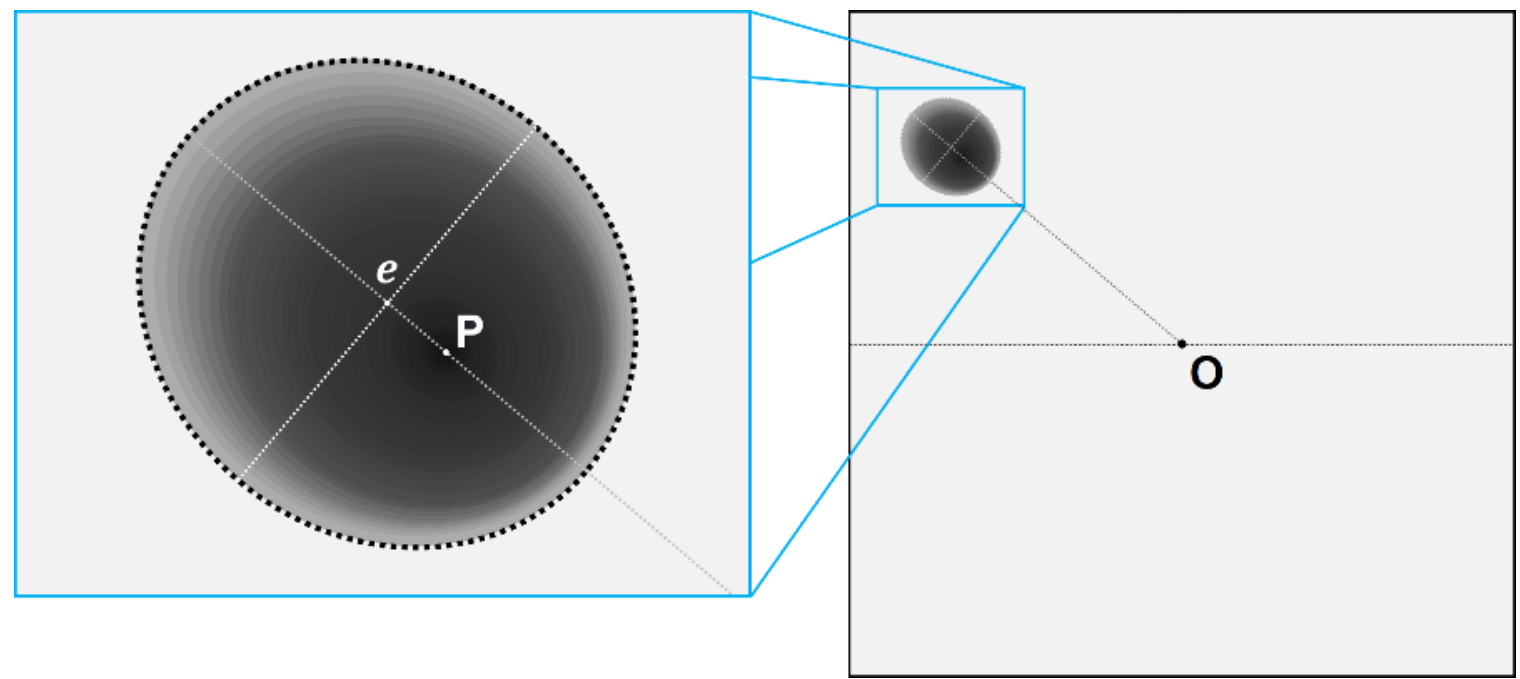

Figure 7. Comparison of the center of an ellipse fit to the outer edge of the projected sphere $\boldsymbol{e}$ to the actual pixel coordinate of the center projection $\mathbf{P}$. The relative positions of $\boldsymbol{e}$ and $\mathbf{P}$ on the imaged elliptical disk in this figure are exaggerated for visualization.

\section{Center projection errors: observed vs. exact}

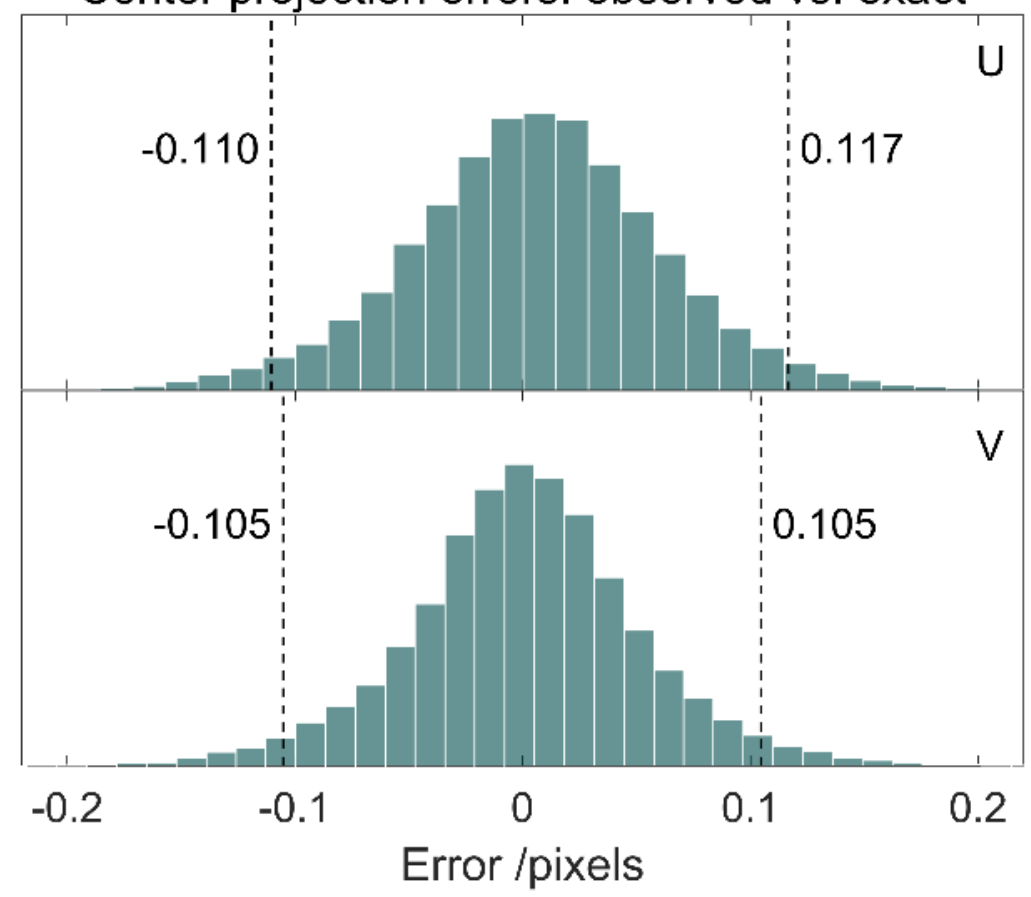

Figure 8. Histograms of error between exact and observed center projection coordinates for simulation 1 . The vertical dashed lines correspond to the upper and lower boundaries of the $95 \%$ error interval. 


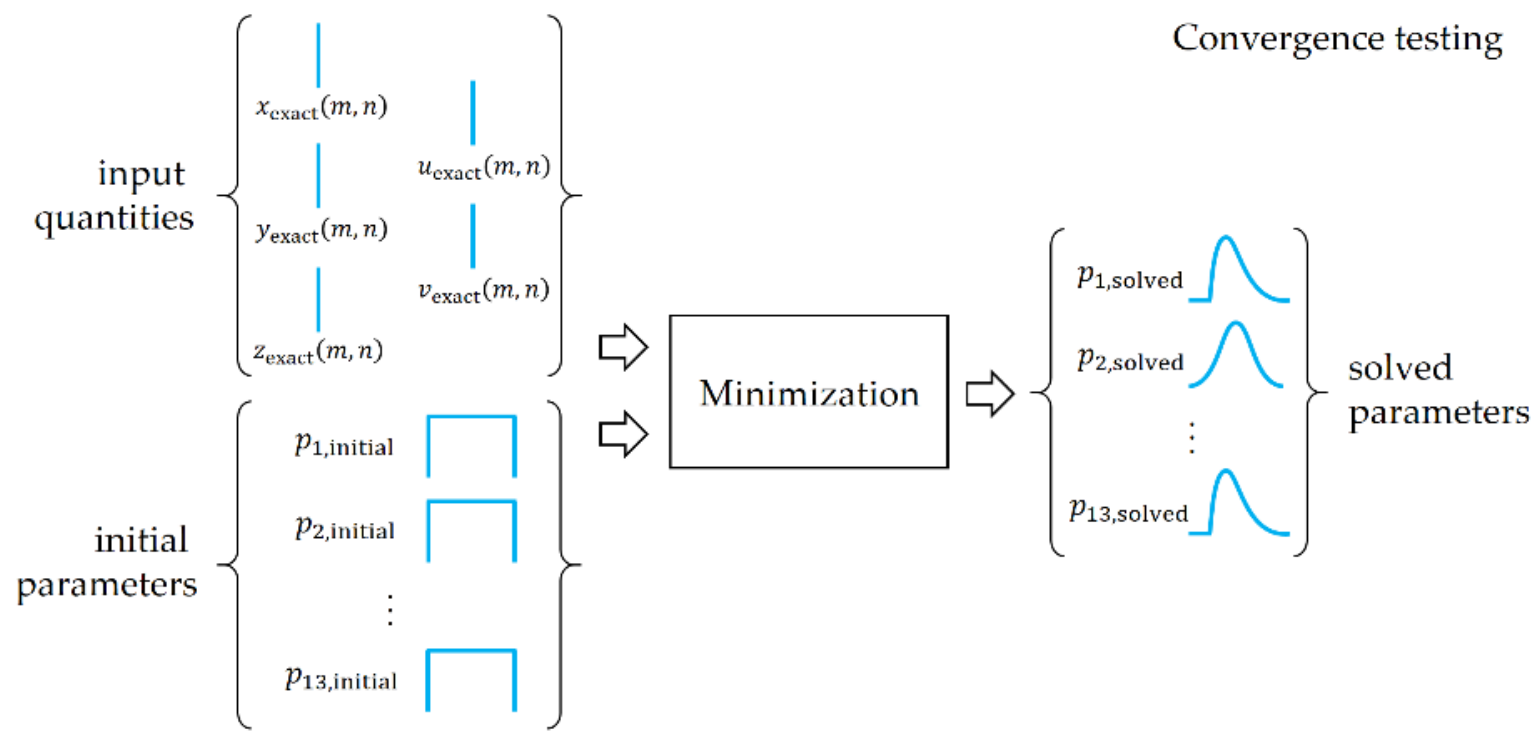

Figure 9. The global optimization technique is tested to ensure consistent convergence irrespective of initial values of the solvable parameters. The input quantities, i.e. sphere center coordinates and center projection coordinates, were set to their exact values for convergence testing.

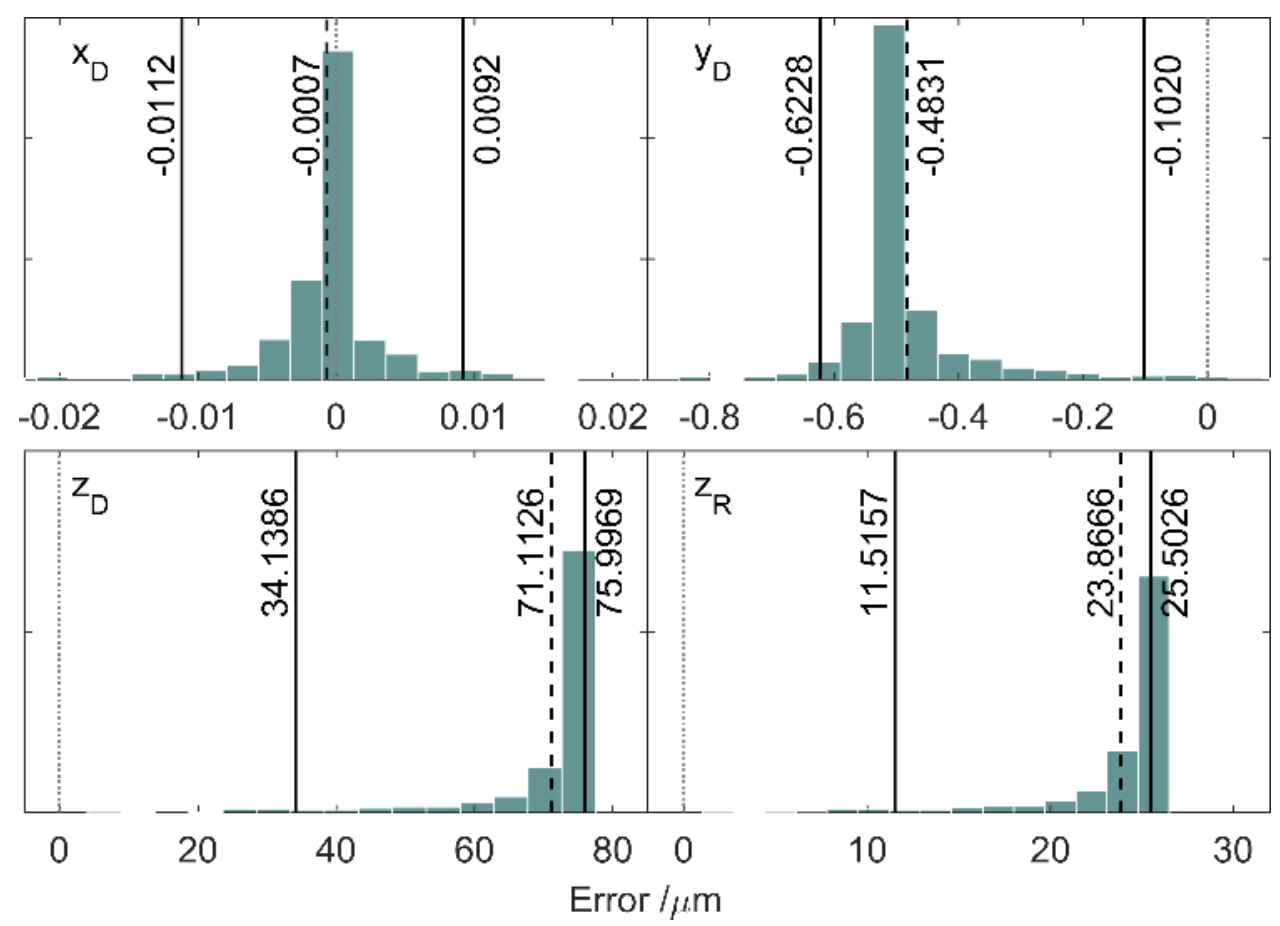

Figure 10. Histograms of solved instrument positional parameters from convergence testing for simulation 1 . The dotted line corresponds to the actual simulated value (zero error) while the solid lines correspond to the lower and upper quantiles for $95 \%$ of the solved parameter values. Dashed lines correspond to the mean of the solved values. 


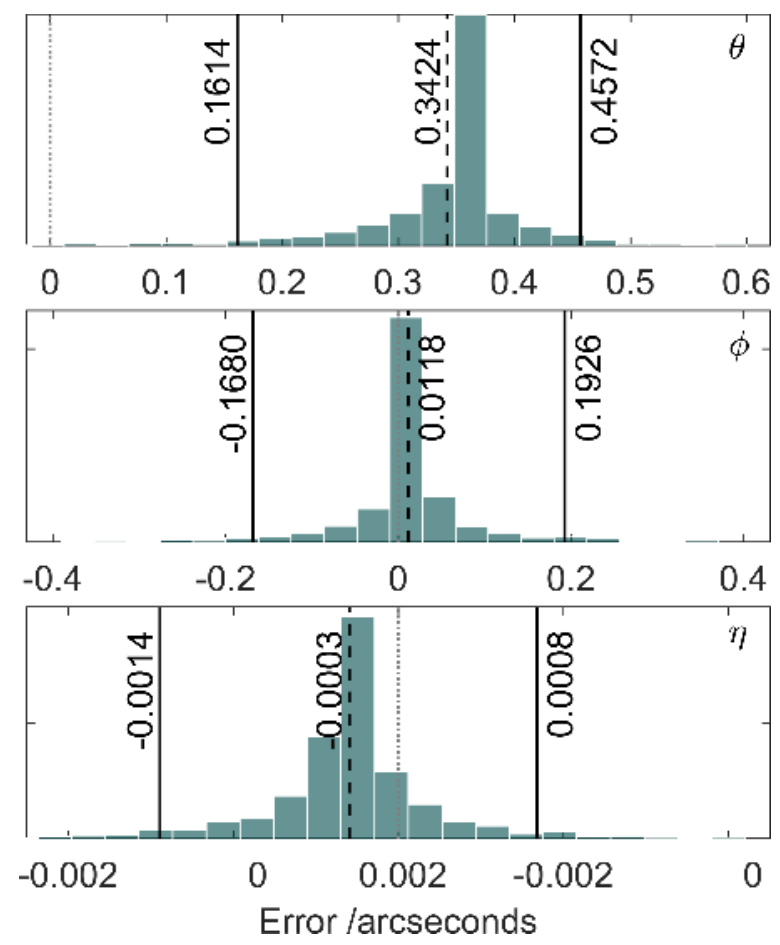

Figure 11. Histograms of solved instrument rotational parameters from convergence testing for simulation 1 . The dotted line corresponds to the actual simulated value (zero error) while the solid lines correspond to the lower and upper quantiles for $95 \%$ of the solved parameter values. Dashed lines correspond to the mean of the solved values.
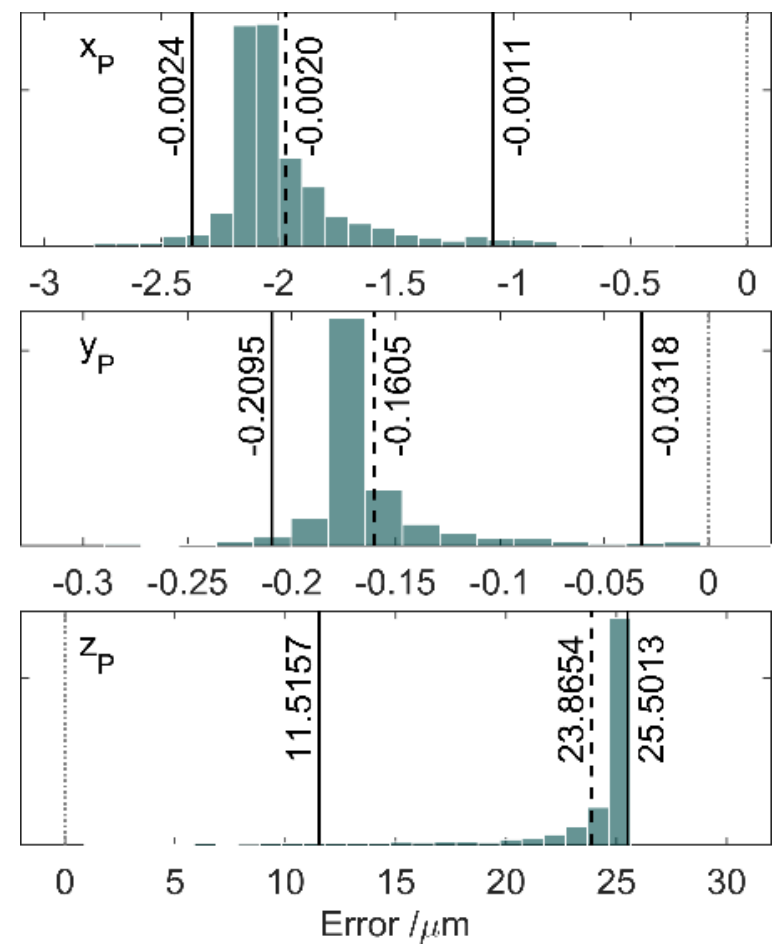

Figure 12. Histograms of solved reference object position from convergence testing for simulation 1 . The dotted line corresponds to the actual simulated value (zero error) while the solid lines correspond to the lower and upper quantiles for $95 \%$ of the solved parameter values. Dashed lines correspond to the mean of the solved values. 


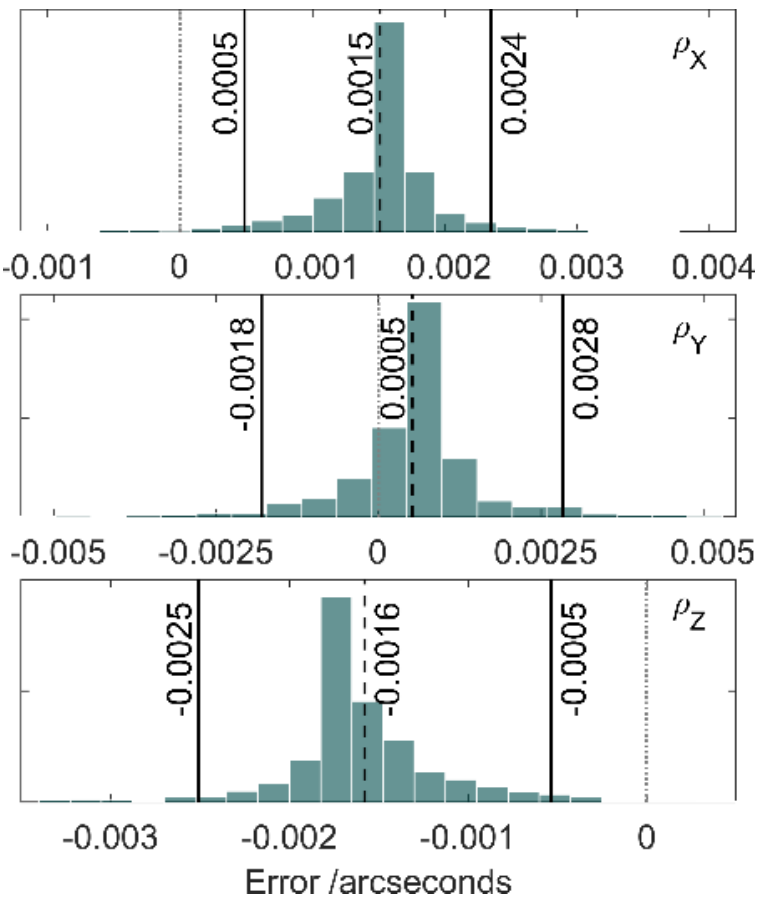

Figure 13. Histograms of solved reference object orientation from convergence testing for simulation 1 . The dotted line corresponds to the actual simulated value (zero error) while the solid lines correspond to the lower and upper quantiles for $95 \%$ of the solved parameter values. Dashed lines correspond to the mean of the solved values.
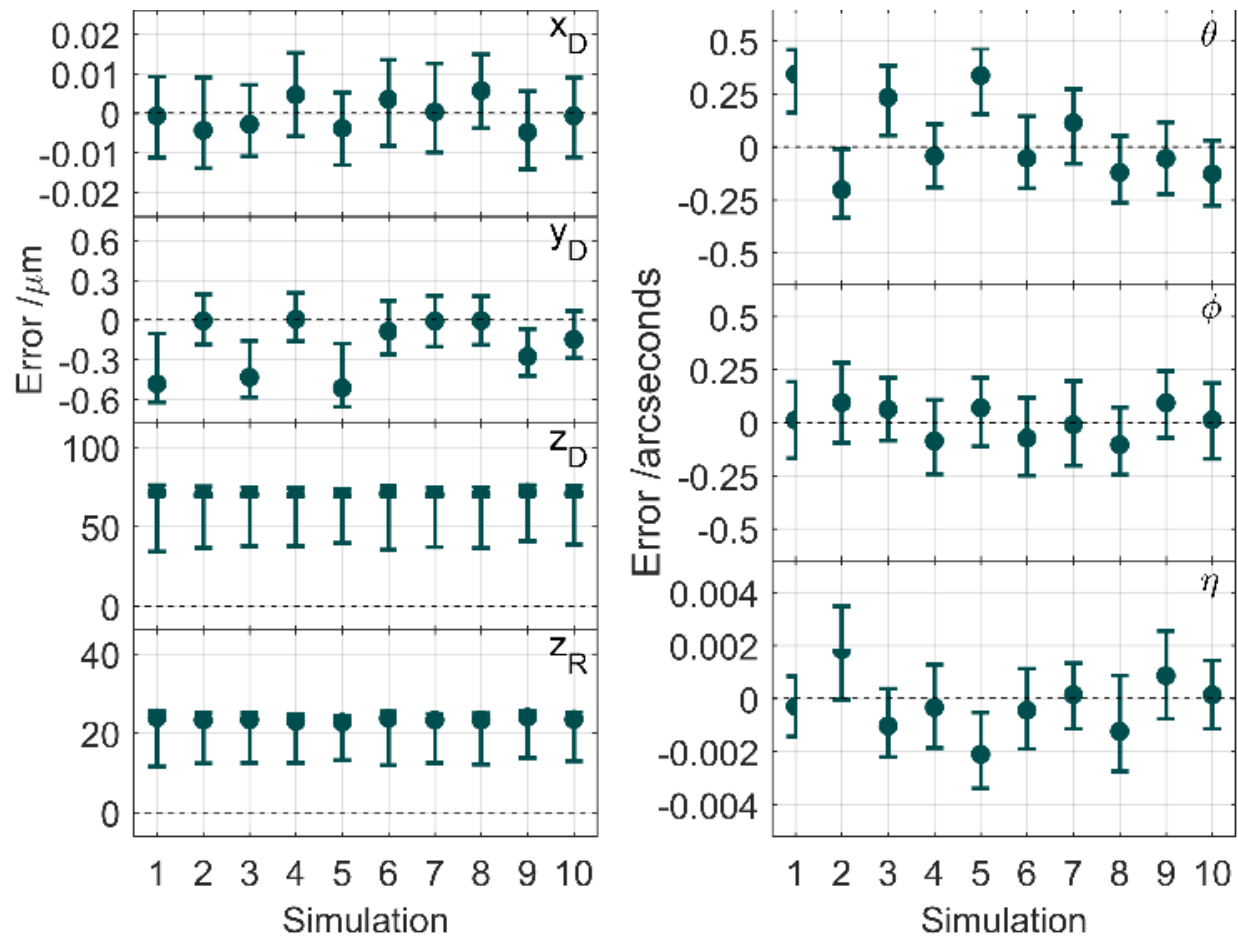

Figure 14. Errors in solved instrument geometrical parameters from convergence testing for all simulations. The circular marker denotes the mean error over 1000 repeat convergence runs, while the error bars correspond to $95 \%$ of the solved parameter values, given by the $2.5 \%$ (bottom) and $97.5 \%$ (top) quantiles. 

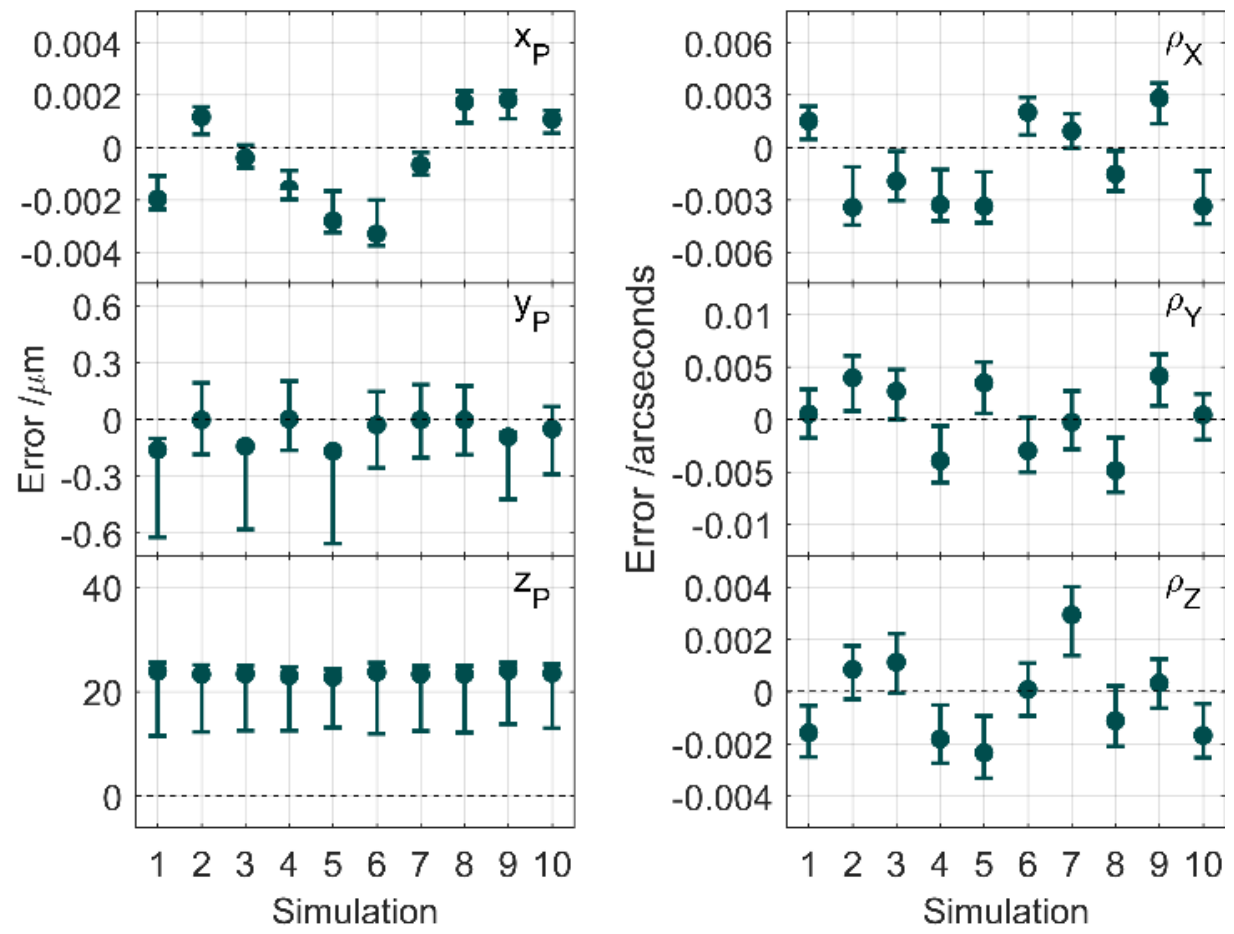

Figure 15. Errors in solved reference object geometrical parameters from convergence testing from all simulations. The circular marker denotes the mean error over 1000 repeat convergence runs, while the error bars correspond to $95 \%$ of the solved parameter values, given by the $2.5 \%$ (bottom) and $97.5 \%$ (top) quantiles.

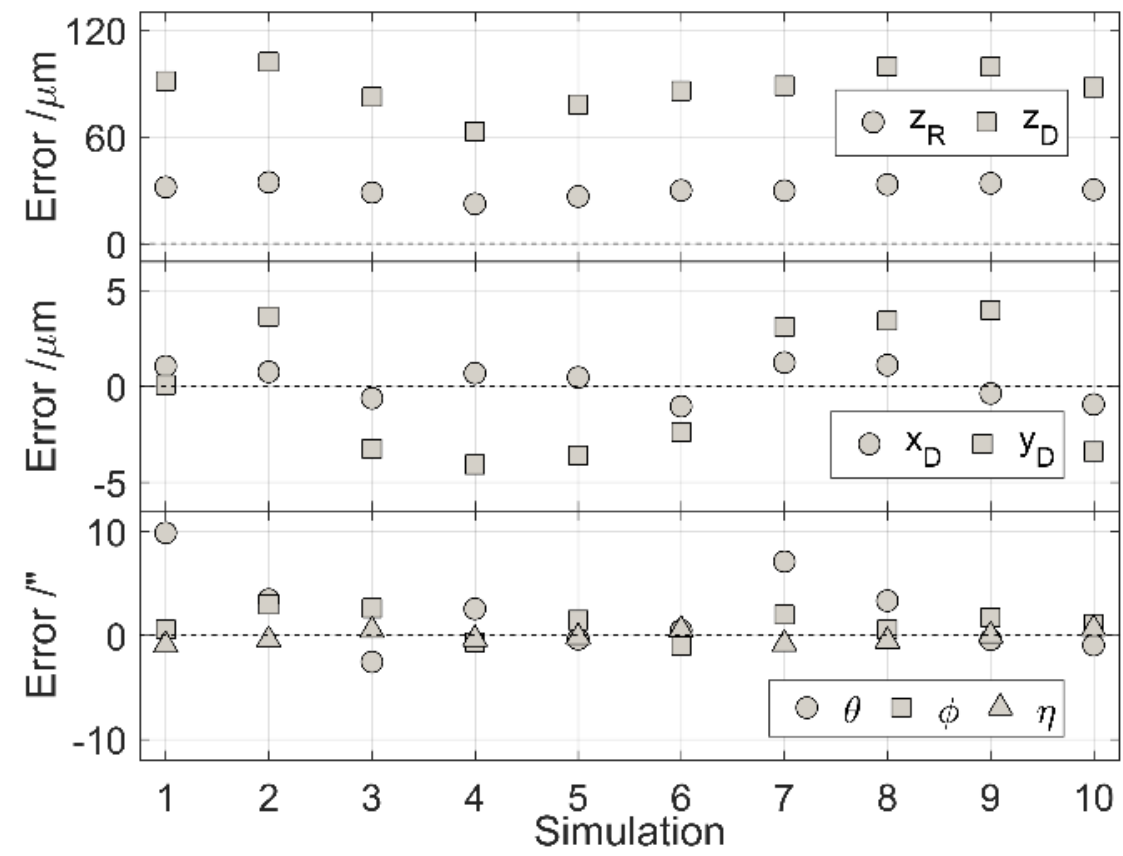

Figure 16. Errors in solved instrument geometrical parameters. 
(C) 2018. This manuscript version is made available under the CC-BY-NC-ND 4.0 license

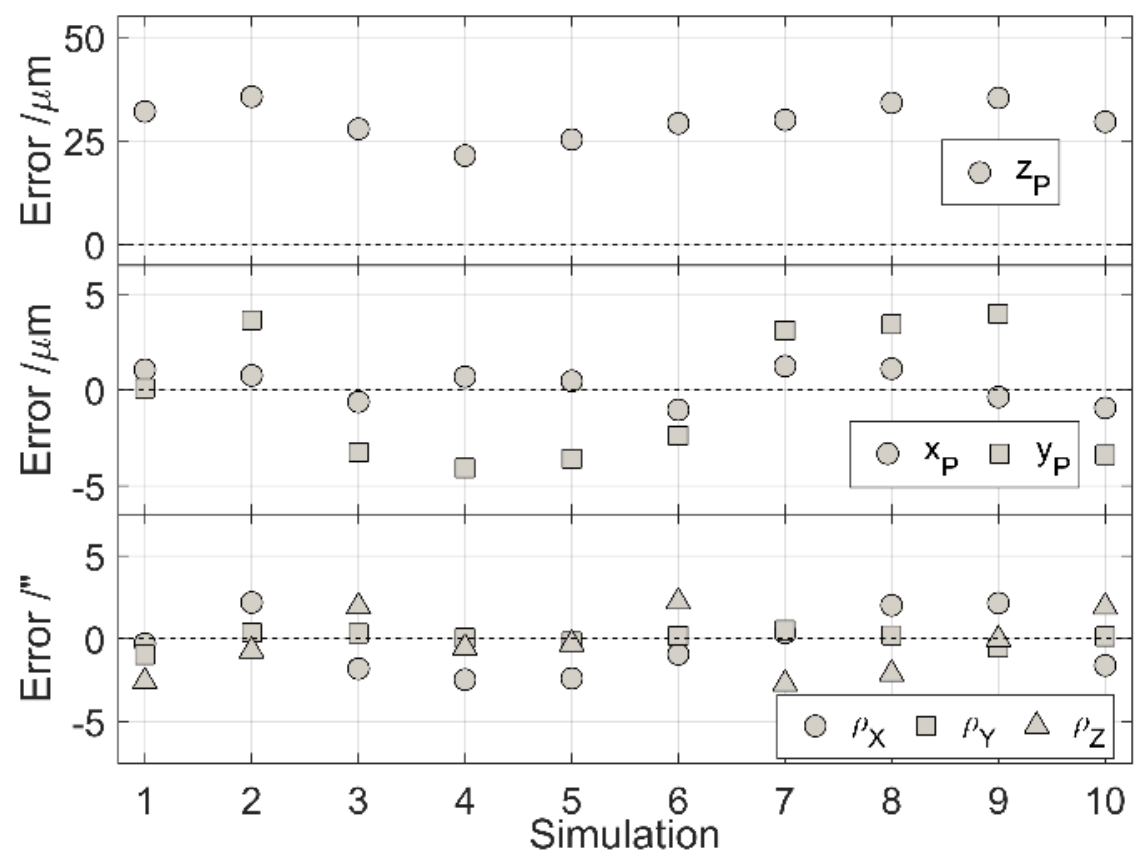

Figure 17. Errors in solved reference object geometrical parameters.

Center projection errors: observed vs. minimized

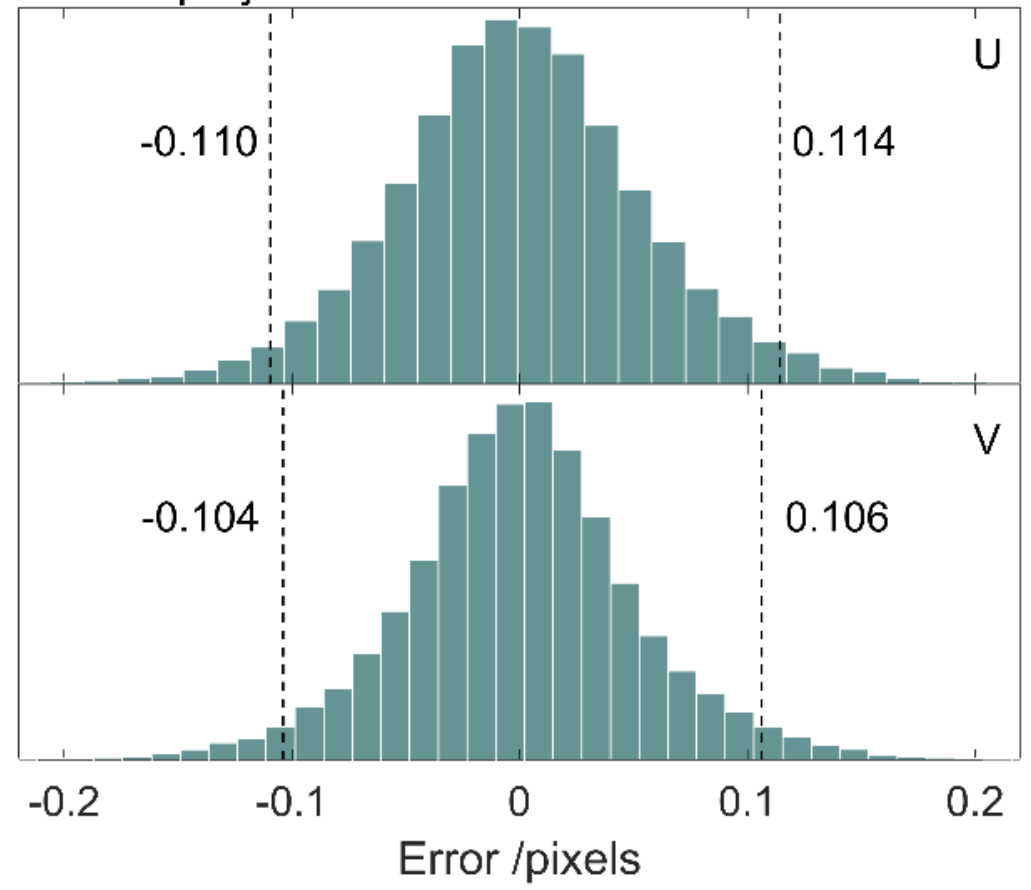

Figure 18. Histograms of error between observed and minimized center projection coordinates for one minimization run on the simulation 1 dataset. The vertical dashed lines correspond to the upper and lower boundaries of $95 \%$ error interval. 


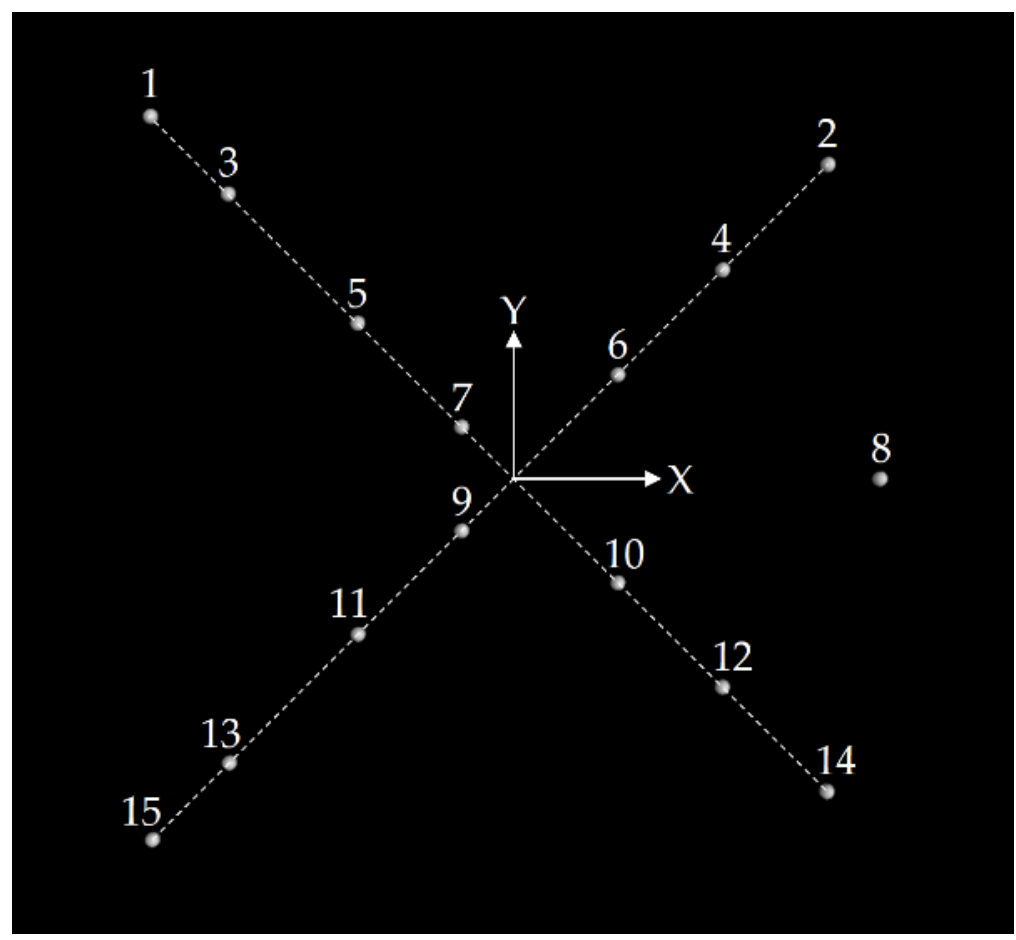

Figure 19. Test object for evaluating the correction of the CT instrument geometry from the output of the proposed geometrical measurement procedure.

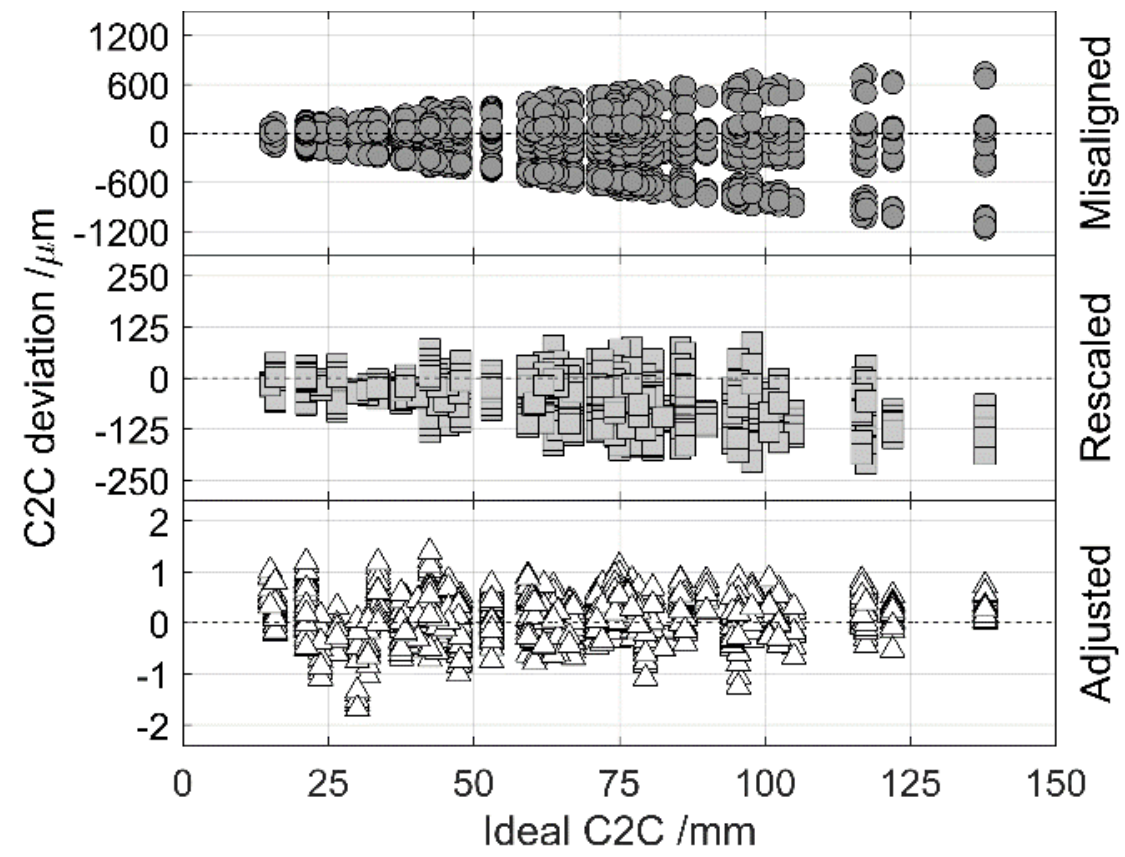

Figure 20. Center-to-center distance deviation from aligned acquisition for nominal, rescaled, and adjusted datasets. 
(C) 2018. This manuscript version is made available under the CC-BY-NC-ND 4.0 license

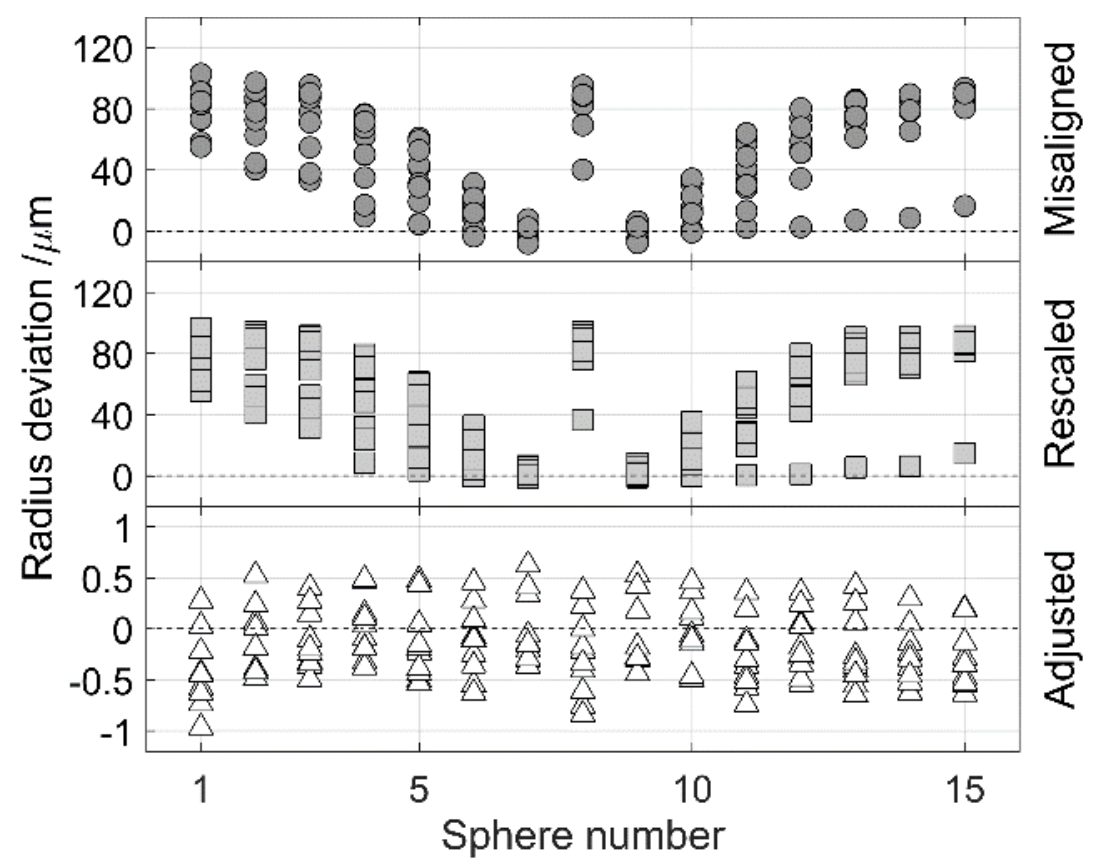

Figure 21. Radius deviation from aligned acquisition for nominal, rescaled, and adjusted datasets. 